\title{
The Expression of Yes-Associated Protein (YAP) Maintains Putative Cancer Stemness and Is Associated with Poor Prognosis in Intrahepatic Cholangiocarcinoma
}

Kensuke Sugiura, Takashi Mishima, Shigetsugu Takano, Hideyuki Yoshitomi, Katsunori Furukawa, Tsukasa Takayashiki, Satoshi Kuboki, Mamoru Takada, Masaru Miyazaki, and Masayuki Ohtsuka

Accepted for publication May 21, 2019.

Address correspondence to Shigetsugu Takano, M.D., Ph.D., or Masayuki Ohtsuka, M.D., Ph.D., Department of General Surgery, Chiba University, Graduate School of Medicine, 1-8-1, Inohana, Chuo-ku, Chiba City, Chiba 260-8677, Japan. E-mail: stakano@faculty.chiba-u.jp or m-otsuka@faculty.chiba-u.jp.

\begin{abstract}
Intrahepatic cholangiocarcinoma (ICC) is resistant to most chemotherapeutic agents. Yes-associated protein (YAP) is related to tumor progression; however, its role in ICC remains unknown. We investigated the mechanism underlying YAP-mediated cancer progression by focusing on the property of cancer stem cells (CSCs) in ICC. Immunohistochemistry results revealed the positive YAP expression in 37 of 52 resected ICC cases. Those with positive YAP expression showed poor prognosis in Kaplan-Meier analysis $(P=0.023)$. YAP expression was associated with vimentin and the putative CSC marker, hepatic oval cell marker 6 (OV-6). The knockdown of YAP expression using specific siRNAs in ICC cells decreased octamer-binding transcription factor 4 (OCT4) expression in Western blot analyses and OV-6 and CD133 expression in flow cytometry analysis. Verteporfin, a YAP inhibitor, decreased $\mathrm{N}$-cadherin and OCT4 expression in Western blot analyses. In vitro sphere formation and anoikis resistance assays revealed the impairment in CSC property and anoikis resistance in response to the decrease in YAP expression. Verteporfin treatment activated the protein kinase $\mathrm{B} /$ mechanistic target of rapamycin signaling pathway and dramatically impaired IL-6-stimulated STAT3 phosphorylation in ICC cells. The combination of verteporfin and rapamycin, an inhibitor of mechanistic target of rapamycin phosphorylation, inhibited cell proliferation and tumor growth. In conclusion, verteporfin regulates multiple signaling pathways and, in combination with rapamycin, might be a promising therapeutic strategy for ICC treatment. (Am J Pathol 2019, 189: 1863-1877; https://doi.org/10.1016/j.ajpath.2019.05.014)
\end{abstract}

Intrahepatic cholangiocarcinoma (ICC) is the second most frequent primary liver cancer after hepatocellular carcinoma and originates from the epithelium of the intrahepatic bile duct. ${ }^{1,2}$ Surgical resection is the first-line treatment for ICC. However, most patients with ICC are diagnosed at the advanced stage. The resectable rate of ICC is only $15 \%$, whereas the median survival time is $\leq 3$ years. ${ }^{3}$ Most ICC cases are resistant to chemotherapeutic agents. ${ }^{4}$ Hence, it is crucial to elucidate the mechanism underlying ICC progression and discover novel therapeutic targets for ICC treatment.

Yes-associated protein (YAP) is a major downstream effector of the Hippo signaling pathway. YAP plays important roles in the regulation of organ size and tissue development, regeneration, and differentiation; and it is associated with the self-renewal ability of stem cells in normal tissues. ${ }^{5-8}$ Activation of the Hippo pathway induces the inactivation of YAP through the phosphorylation of Ser127 by a large tumor suppressor kinase 1/2. The

Supported by the grant in aid for scientific research (KAKENHI): KIBAN C: 19K09190 (T.M., S.T., M.O.), KIBAN B: 19H03725 (S.T., H.Y., M.O.), Challenge Exploratory Research: 16K15607 (S.T., H.Y., M.M.), KIBAN C: 18K08671 (H.Y., S.T., M.O.), Young Scientists: 19 K16824 (M.T.), KIBAN C: 17K10661 (S.K., M.O.), KIBAN B: 17 H04287 (M.M., S.T., H.Y., M.O.), and KIBAN C: 16K10589 (M.O., S.T., S.K.); the Kashiwado Memorial Foundation (S.T.); and the Japanese Foundation for Multidisciplinary Treatment of Cancer (S.T.).

Disclosures: None declared. 

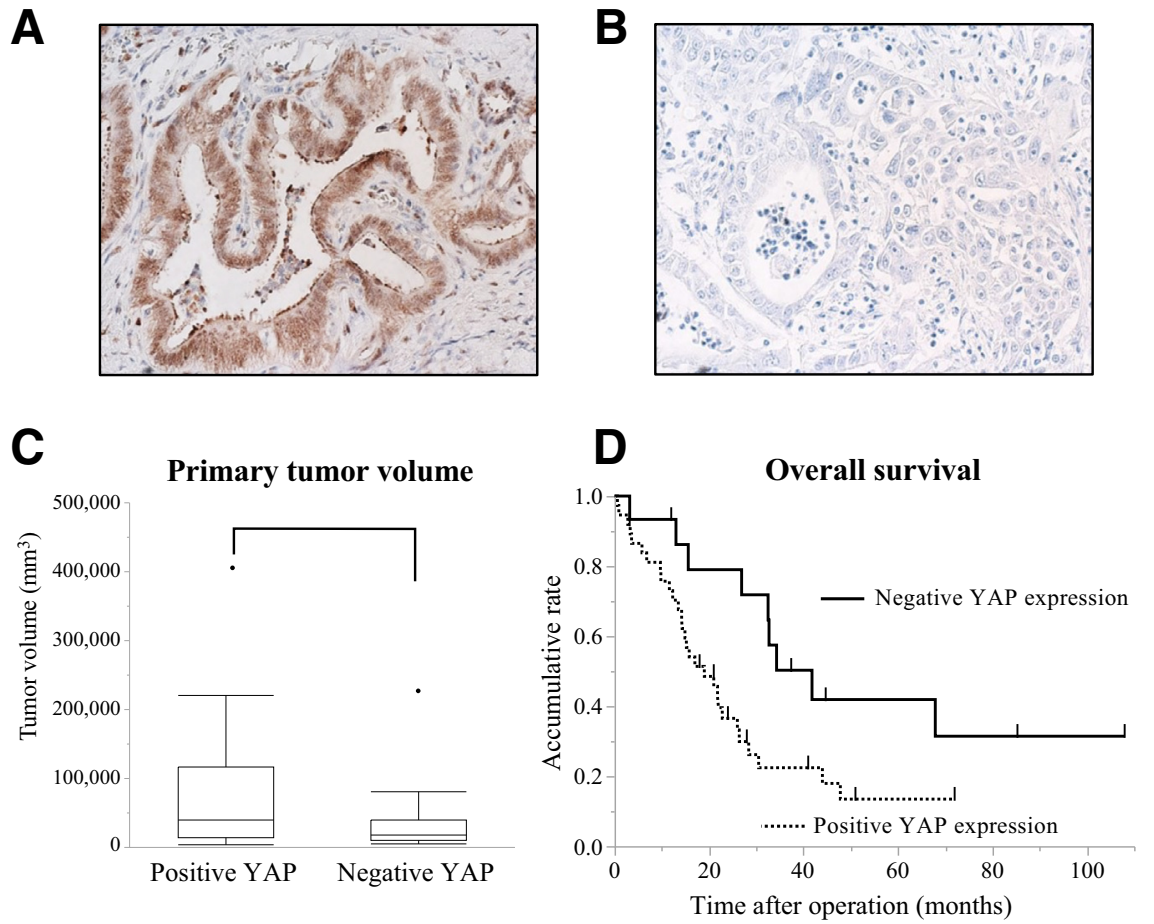

Figure 1 Immunohistochemistry of YAP expression in intrahepatic cholangiocarcinoma (ICC). Positive YAP expression predicts poor prognosis of patients with ICC after curative resection. A: Positive YAP expression in primary ICC. YAP is primarily expressed in the nucleus of ICC cells. B: Negative YAP expression in primary ICC. C: Comparison of primary tumor volume between groups with positive YAP expression and negative YAP expression. The box-plot analysis shows significantly larger tumor volumes in the positive YAP expression group than in the negative YAP expression groups ( $P=0.038, U$-test). D: Kaplan-Meier analysis for overall survival of patients based on the positive/negative YAP expression in ICC tissues. Positive YAP expression group shows significantly worse outcomes than the negative YAP expression group $(P=0.023$, log-rank test). $n=37$ (A); $n=15$ (B). Original magnification, $\times 200(\mathbf{A}$ and $\mathbf{B})$. phosphorylated YAP is bound by a 14-3-3 protein in the cytoplasm. ${ }^{9}$ On the contrary, the translocation of the unphosphorylated YAP into the nucleus leads to its binding to the TEA domain (TEAD) family of transcription factors, in turn leading to cell proliferation and apoptosis inhibition in normal tissues. ${ }^{9}$

The functions of YAP have been observed in various malignancies, such as hepatocellular carcinoma, ${ }^{10}$ ovarian cancer, ${ }^{11}$ colorectal cancer, ${ }^{12}$ gastric cancer, ${ }^{13}$ and gallbladder cancer. ${ }^{14}$ YAP overexpression is associated with angiogenesis, invasion, and epithelial-to-mesenchymal transition (EMT) in malignancies, including ICC, resulting in an unfavorable prognosis. ${ }^{15-17}$ A premalignant lesion in the liver tumor is stimulated by bile acid, and YAP and the upstream target IQ motif-containing GTPase-activating protein 1 are related to bile acid-mediated regulation of liver growth and tumorigenesis via the Hippo pathway. ${ }^{18}$

Putative cancer stem cells (CSCs) exhibit self-renewal capacity and the ability of multilineage differentiation in neoplastic tumors. ${ }^{19}$ The treatment of CSCs may serve as a novel targeting therapy because CSCs are resistant to various therapeutic agents that may result in tumor relapse after chemoradiotherapy or surgical resection. Recent studies have demonstrated that YAP maintains the CSC-like property in urinary bladder cancer ${ }^{20}$ and breast cancer. ${ }^{21}$ However, its role in ICC remains unclear.

Herein, we describe the clinical significance of YAP expression by focusing on the relationship between YAP expression and putative CSC property in ICC. The inhibition of YAP activation resulted in the impairment in CSC property, proliferation ability of ICC in vitro, and tumor growth in vivo. The data provide insights into effects of YAP on CSC property and a new platform for translational therapeutics in ICC.

\section{Materials and Methods}

\section{Patient Samples and Ethics Approval}

The clinical samples and data were obtained from 52 patients who underwent curative surgical resection for the mass-forming type of ICC in the Department of General Surgery, Chiba University Hospital (Chiba, Japan) from April 2004 to June 2016. All patients were histologically diagnosed with primary ICC. Nine patients were administrated chemotherapy before surgery, and 16 patients were administrated adjuvant chemotherapy after surgery. The Ethics Committees of our institute approved the protocol of this study (approval number 2965), and written informed consent was obtained from each patient before surgery.

\section{Immunohistochemistry}

Slides were deparaffinized in xylene and rehydrated through a graded alcohol series. For antigen retrieval, the slides were microwaved in $10 \mathrm{mmol} / \mathrm{L}$ citrate buffer $(\mathrm{pH} 6.0)$ for 12 minutes. Endogenous peroxidase activity was blocked with $3 \% \mathrm{H}_{2} \mathrm{O}_{2}$ in methanol. After blocking any non-specific protein binding, the fixed tissues were incubated overnight at $4^{\circ} \mathrm{C}$ with primary antibodies specific for YAP (Santa Cruz Biotechnology, Inc., Dallas, TX; dilution 1:1000), E-cadherin (Cell Signaling Technology, Beverly, MA; dilution 
Table 1 Relationships between YAP Expression and Clinicopathologic Features of Patients with ICC

\begin{tabular}{|c|c|c|c|}
\hline \multirow[b]{2}{*}{ Feature } & \multicolumn{2}{|l|}{ YAP expression } & \multirow[b]{2}{*}{$P$ value } \\
\hline & Positive $(n=37)$ & Negative $(n=15)$ & \\
\hline Age in years, means $\pm S D$ & $66.4 \pm 8.7$ & $64.3 \pm 8.4$ & 0.70 \\
\hline Serum CEA $(\geq 5 /<5), \mathrm{ng} / \mathrm{mL}$ & $5: 32$ & $2: 13$ & 0.66 \\
\hline Serum CA19-9 $(\geq 36.8 /<36.8), \mathrm{U} / \mathrm{mL}$ & $17: 20$ & $8: 7$ & 0.40 \\
\hline Primary tumor volume $(\geq 34,000 /<34,000), \mathrm{mm}^{3}$ & $25: 12$ & $5: 10$ & $0.02^{*}$ \\
\hline Differentiation $\left(\mathrm{G}_{1} / \mathrm{G}_{2} \text { and } \mathrm{G}_{3}\right)^{\dagger}$ & $27: 10$ & $14: 1$ & 0.20 \\
\hline ICGR15, means \pm SD, \% & $9.0 \pm 5.4$ & $10.0 \pm 5.3$ & 0.14 \\
\hline Operation method (<lobectomy/ $\geq$ lobectomy) & $14: 23$ & $5: 10$ & 0.76 \\
\hline $\mathrm{T}$ factor $(1 / 2 \mathrm{a} / 2 \mathrm{~b} / 3 / 4)^{\dagger}$ & $2: 7: 3: 2: 23$ & $1: 2: 1: 3: 8$ & 0.82 \\
\hline $\mathrm{N}$ factor $(0 / 1)^{\dagger}$ & $18: 19$ & $10: 5$ & 0.23 \\
\hline
\end{tabular}

$* P<0.05$.

${ }^{\dagger}$ According to the International Union Against Cancer Classification.

CA, carbohydrate antigen; CEA, carcinoembryonic antigen; ICC, intrahepatic cholangiocarcinoma; ICGR, indocyanine green retention; NS, not significant.

1:400), vimentin (Santa Cruz Biotechnology, Inc.; dilution 1:100), matrix metalloproteinase (MMP)-9 (Santa Cruz Biotechnology, Inc.; dilution 1:100), OV-6 (R\&D Systems, Minneapolis, MN; dilution 1:100), or phosphorylated STAT3 (p-STAT3; Cell Signaling Technology; dilution 1:100). The slides were washed three times with phosphatebuffered saline (PBS) and treated with a biotinylated secondary antibody for 30 minutes at room temperature. An EnVision kit (Dako, Glostrup, Denmark) and 3,3'-diaminobenzidine chromogen were used for visualization. Immunohistochemistry (IHC) staining was evaluated and independently scored by two pathologists (K.S. and T.M.) blinded to the clinical characteristics of the patients. YAP expression in the nucleus was evaluated and categorized into two groups (positive and negative expression). IHC staining for E-cadherin (membranous staining of cancer cells), vimentin (cytoplasm staining of cancer cells), MMP9 (nucleus staining of cancer cells), and OV-6 (cytoplasm staining of cancer cells) was also evaluated. The staining patterns of these markers were scored as low expression $(0 \%$ to $30 \%$ of the cancer cells had positive staining) or high expression ( $>30 \%$ of the tumor cells had positive staining).

\section{Cell Culture}

The human ICC cell lines HuCCT1 and HuH-28 were obtained from the Japanese Collection of Research Bioresources Cell Bank. TKKK cells were provided by the Cell Resource Center for Biomedical Research, Institute of Development, Aging and Cancer, Tohoku University (Sendai, Japan). HuCCT1 and HuH-28 cells were cultured in RPMI 1640 medium (Thermo Fisher Scientific, Waltham,

Table 2 Univariate Analysis and Multivariate Analysis Using the Cox Proportional Hazard Model

\begin{tabular}{|c|c|c|c|c|}
\hline Variable & \multicolumn{2}{|l|}{ Univariate analysis } & \multicolumn{2}{|l|}{ Multivariate analysis } \\
\hline Age $(\geq 70 /<70$ years $)$ & $1.59(1.30-5.85)$ & 0.18 & & \\
\hline Serum CEA $(\geq 5 /<5), \mathrm{ng} / \mathrm{mL}$ & $1.21(0.45-2.70)$ & 0.67 & & \\
\hline Serum CA19-9 $(\geq 36.8 /<36.8), U / m L$ & $2.25(1.11-4.60)$ & 0.02 & $1.38(0.63-2.98)$ & 0.40 \\
\hline Primary tumor volume $(\geq 34,000 /<34,000), \mathrm{mm}^{3}$ & $2.63(1.30-5.85)$ & 0.006 & $1.57(0.68-3.74)$ & 0.28 \\
\hline Vascular invasion $(+/-)$ & $1.31(0.63-3.09)$ & 0.48 & & \\
\hline Differentiation $\left(\mathrm{G}_{1} / \mathrm{G}_{2} \text { and } \mathrm{G}_{3}\right)^{*}$ & $2.49(1.12-5.11)$ & 0.02 & $2.39(1.01-5.28)$ & 0.04 \\
\hline T factor $(1 / 2 a / 2 b \text { and } 3 / 4)^{*}$ & $1.41(0.70-3.07)$ & 0.33 & & \\
\hline$N$ factor $(0 / 1)^{*}$ & $1.84(0.96-3.53)$ & 0.06 & $1.60(0.67-3.81)$ & 0.28 \\
\hline Para-aortic lymph node metastasis $(+/-)$ & $3.49(1.15-8.64)$ & 0.01 & $1.68(0.48-5.18)$ & 0.39 \\
\hline YAP expression (positive/negative) & $2.58(1.24-5.92)$ & 0.01 & $2.02(0.84-5.12)$ & 0.11 \\
\hline
\end{tabular}

${ }^{*}$ According to the International Union Against Cancer Classification.

CA, carbohydrate antigen; CEA, carcinoembryonic antigen; HR, hazard ratio. 

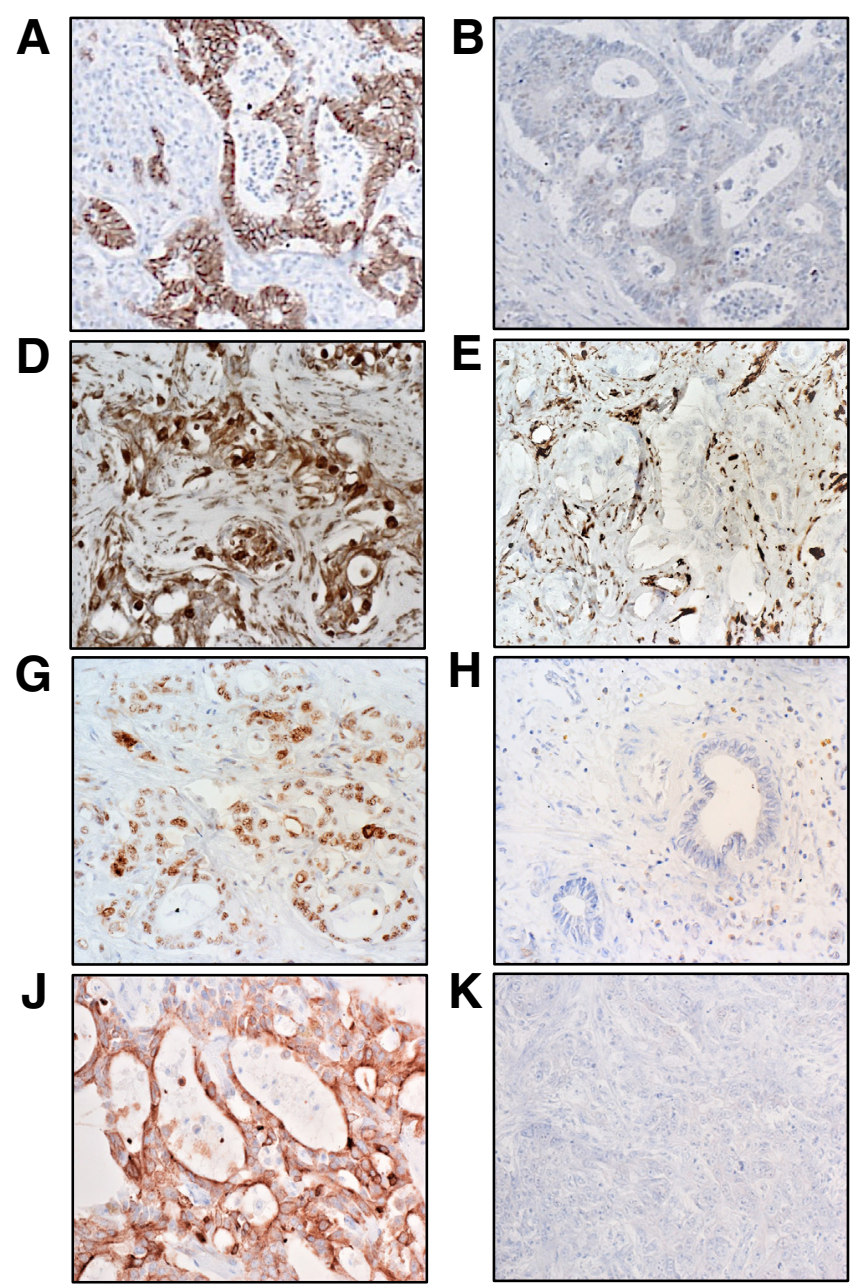
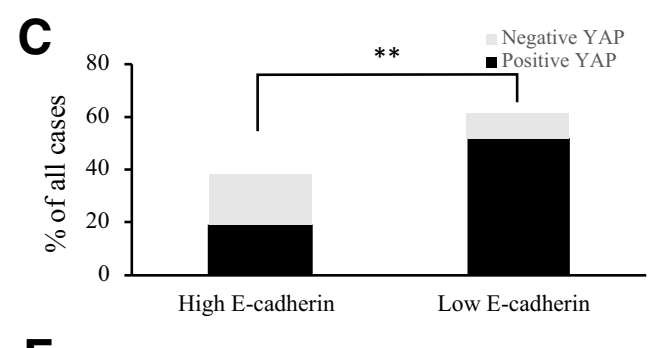

F
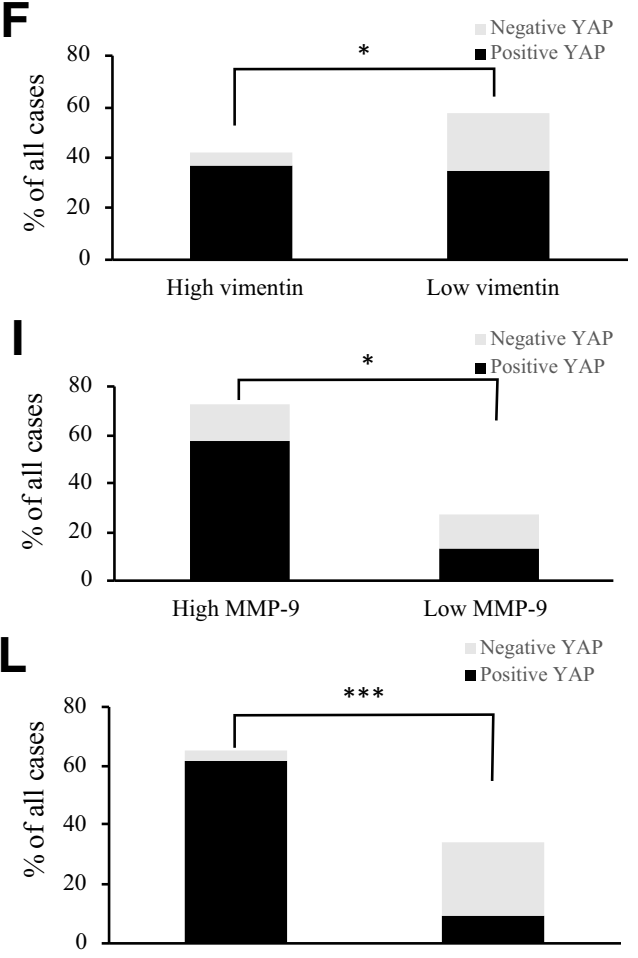

High OV-6

Low OV-6

Figure 2 Immunohistochemical (IHC) analysis and relationship between YAP and epithelial-to-mesenchymal transition/cancer stem cell markers in human intrahepatic cholangiocarcinoma (ICC) tissues. Staining intensity of E-cadherin (A and $\mathbf{B})$, vimentin (D and $\mathbf{E})$, matrix metallopeptidase (MMP)-9 (G and $\mathbf{H})$, and OV-6 (J and $\mathbf{K})$ in primary ICC tissue was evaluated and categorized into two groups [high expression $(\mathbf{A}, \mathbf{D}, \mathbf{G}$, and $\mathbf{J})$ and low expression $(\mathbf{B}, \mathbf{E}, \mathbf{H}$, and $\mathbf{K})$ ]. Comparison of IHC staining intensity for YAP and E-cadherin (C), vimentin (F), MMP-9 (I), and OV-6 (L) in primary ICC tissues. High E-cadherin expression (A), low E-cadherin expression (B), and negative correlation between YAP and E-cadherin expression (C) are shown. High vimentin expression (D), low vimentin expression (E), and positive correlation between YAP and vimentin expression (F) are shown. High MMP-9 expression (G), low MMP-9 expression (H), and positive correlation between YAP and MMP-9 expression (I) are shown. High 0V-6 expression (J), low 0V-6 expression (K), and strong positive correlation between YAP and OV-6 expression (L) are shown. $\chi^{2}$ Test was used for statistical analyses. $n=20(\mathbf{A}) ; n=32(\mathbf{B}) ; n=21(\mathbf{D}) ; n=31(\mathbf{E}) ; n=38(\mathbf{G}) ;$ $n=14(\mathbf{H}) ; n=34(\mathbf{J}) ; n=18(\mathbf{K}) .{ }^{*} P<0.05,{ }^{*} P P<0.01$, and ${ }^{* * *} P<0.001$. Original magnification, $\times 200(\mathbf{A}, \mathbf{B}, \mathbf{D}, \mathbf{E}, \mathbf{G}, \mathbf{H}, \mathbf{J}$, and $\mathbf{K})$.

MA) supplemented with $10 \%$ fetal bovine serum. TKKK cells were cultured on a collagen plate (Sumitomo Bakelite Co, Ltd, Tokyo, Japan) in low-glucose Dulbecco's modified Eagle's medium (Sigma-Aldrich, St. Louis, MO) with $10 \%$ fetal bovine serum at $37^{\circ} \mathrm{C}$ and $5 \% \mathrm{CO}_{2}$.

\section{Transfection and Gene Silencing}

Double-stranded siRNAs used to knockdown YAP expression were as follows: siRNA1, Hs_YAP1_6 (catalog number SI04438637); siRNA2, Hs_YAP1_8 (catalog number SI04438651); and control siRNA (AllStars negative-control siRNA; Qiagen Inc., Valencia, CA). These siRNAs (final concentration, $20 \mathrm{nmol} / \mathrm{L}$ ) were transfected into HuCCT1 and TKKK cells using Lipofectamine RNAiMAX (Thermo Fisher Scientific). The cells were harvested 72 hours after transfection for Western blot analysis, sphere formation assay, and anoikis resistance assay.

\section{Western Blot Analysis}

Total cellular proteins were purified with radioimmunoprecipitation assay buffer (Sigma-Aldrich). Protein $(30 \mu \mathrm{g})$ was loaded onto a $7.5 \%$ to $15 \%$ XV PANTERA Gel (DRC; Tama, Tokyo, Japan), and the separated protein bands were transferred onto a polyvinylidene difluoride membrane. The membrane was blocked with $5 \%$ milk and incubated at $4{ }^{\circ} \mathrm{C}$ overnight with the following primary 
A

TKKK HuCCT1 HuH-28

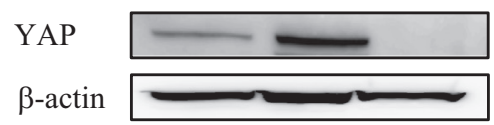

C

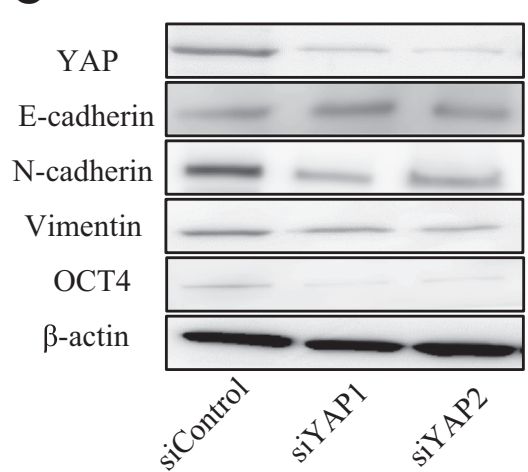

E

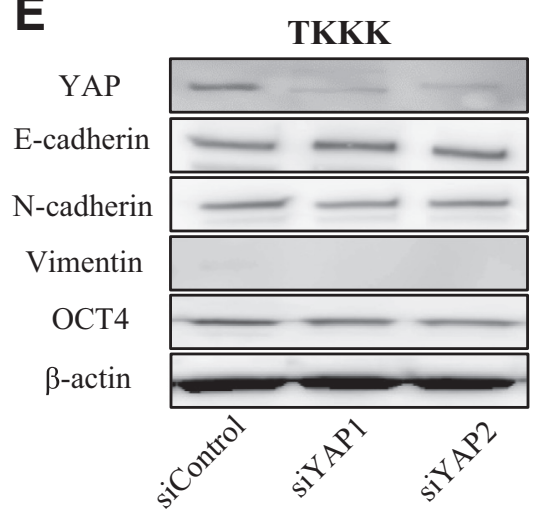

B

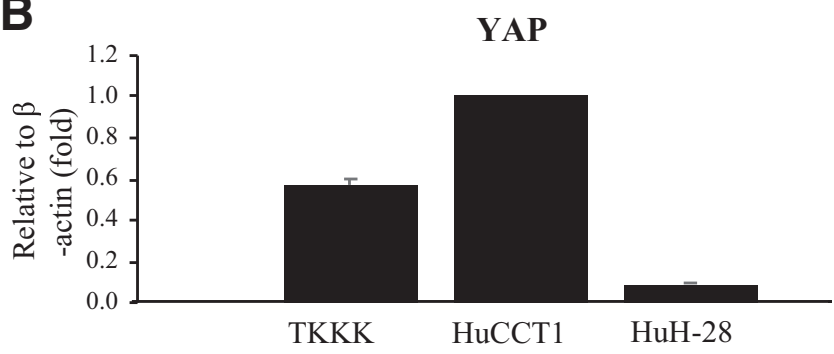

$\mathbf{D}$

HuCCT1

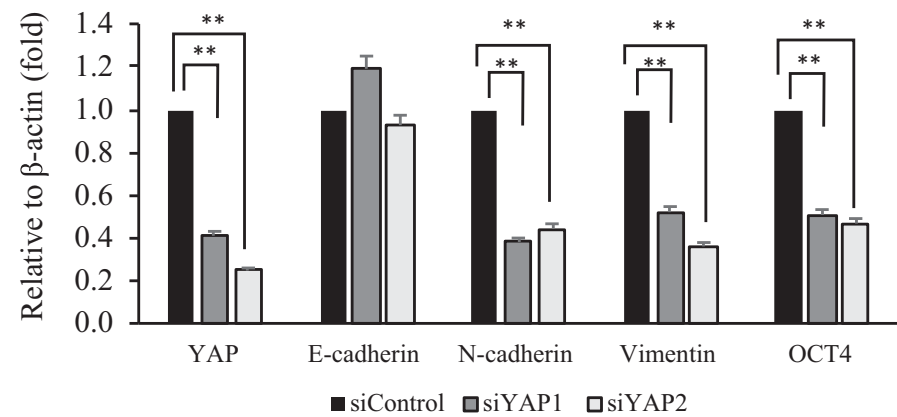

$\mathbf{F}$

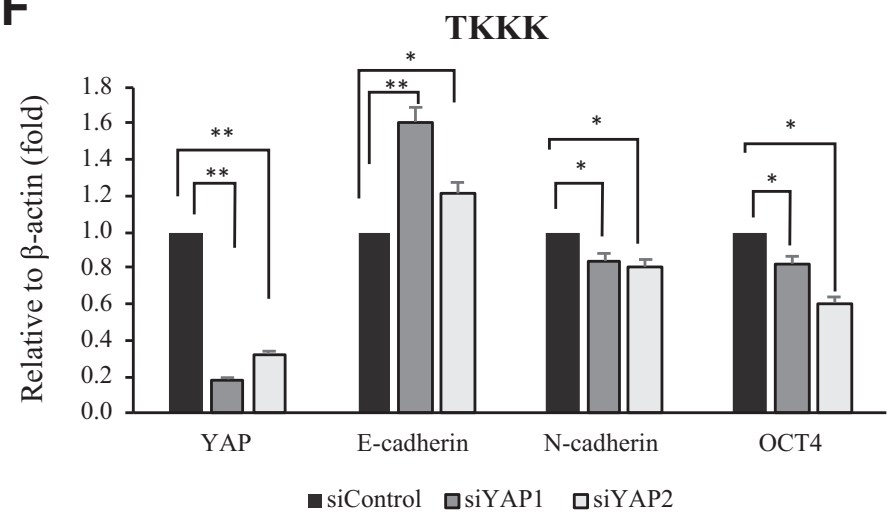

Figure 3 YAP expression is associated with the expression of epithelial-to-mesenchymal transition and cancer stem cell markers in intrahepatic cholangiocarcinoma (ICC) cell lines. A and B: Comparative analysis of YAP expression among three ICC cell lines. A: Western blot analysis. B: Comparison of the band intensities by densitometry among three ICC cell lines. $\mathbf{C}$ and E: Expression levels of YAP, E-cadherin, N-cadherin, vimentin, and OCT4 in HuCCT1 cells (C) and TKKK cells (E) treated with control siRNA (siControl) or YAP-specific siRNAs 1 and 2 (siYAP1 and siYAP2, respectively) by Western blot analysis. D and F: Comparisons of the band intensities of these markers by densitometry in HuCCT1 (D) and TKKK (F) cells. The band intensities were normalized to the intensity of $\beta$-actin. C, D, E, and F: Experiments were repeated three times. Data are expressed as means \pm SEM (B, D, and $\mathbf{F})$. ${ }^{*} P<0.05,{ }^{* *} P<0.01$ (Welch $t$-test).

antibodies: YAP (Santa Cruz Biotechnology, Inc.; dilution 1:1000), phosphorylated YAP (Cell Signaling Technology; dilution 1:1000), E-cadherin (Cell Signaling Technology; dilution 1:1000), N-cadherin (Santa Cruz Biotechnology, Inc.; dilution 1:1000), vimentin (Santa Cruz Biotechnology, Inc.; dilution 1:1000), OV-6 (R\&D Systems; dilution 1:1000), OCT4 (Abcam, Cambridge, UK; dilution 1:1000), Akt (Cell Signaling Technology; dilution 1:1000), phosphorylated Akt (p-Akt; Cell Signaling Technology; dilution 1:1000), mechanistic target of rapamycin (mTOR; Cell Signaling Technology; dilution 1:1000), phosphorylated mTOR (p-mTOR; Cell Signaling Technology; dilution 1:1000), STAT3 (Cell Signaling
Technology; dilution 1:1000), p-STAT3 (Cell Signaling Technology; dilution 1:1000), and $\beta$-actin (Cell Signaling Technology; dilution 1:2000). After washing three times with tris-buffered saline with Tween 20, a horseradish peroxidase-conjugated anti-rabbit antibody (Santa Cruz Biotechnology, Inc.; dilution 1:2000) was added to the membrane as the secondary antibody at room temperature for 1 hour. Protein bands were detected by enhanced chemiluminescence (GE Healthcare, Buckinghamshire, UK), and images were obtained with the LAS 4000 camera system (Fujifilm, Tokyo, Japan). Quantification was performed with ImageJ software version 1.51 (NIH, Bethesda, MD; http://imagej.nih.gov/ij). 
A

\section{HuCCT1}
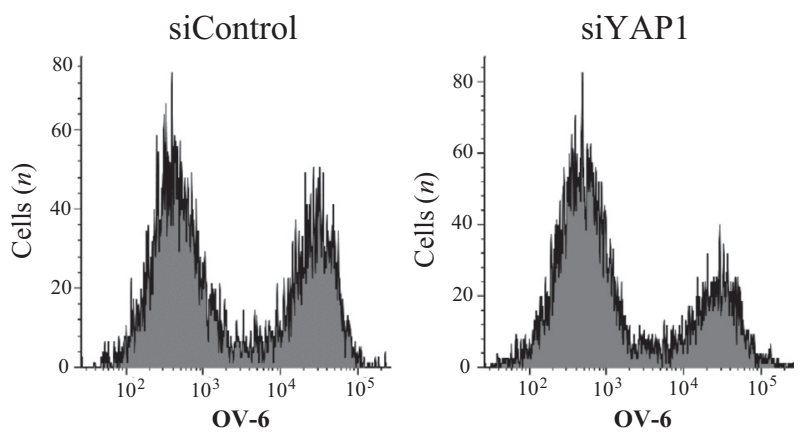

B

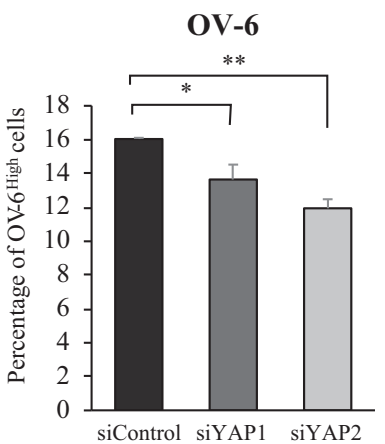

C

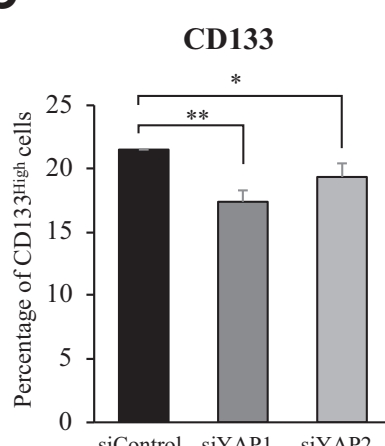

Figure 4 Analyses of the expression between YAP and cancer stem cell markers by flow cytometry. A: Flow cytometry analysis for OV-6 expression in HuCCT1 cells treated with control siRNA (siControl) or YAP-specific siRNA 1 (siYAP1). B: Percentage of subpopulation of OV- $6^{\text {High }}$ cells in HuCCT1 cells treated with control or two siYAPs (control, $16.0 \%$; siYAP1, 13.6\%; siYAP2, $11.9 \%$ ). C: Percentage of subpopulation of CD133 ${ }^{\text {High }}$ cells in HuCCT1 cells treated with control or two siYAPs (control, 21.5\%; siYAP1, 17.4\%; siYAP2, 19.4\%). All experiments were repeated at least three times. Data are expressed as means $\pm \mathrm{SEM}$ (B and $\mathbf{C}$ ). ${ }^{*} P<0.05,{ }^{*} * P<0.01$ (Welch $t$ test).

Flow Cytometry Analysis by Fluorescence-Activated Cell Sorting

One million cells were suspended in $100 \mu \mathrm{L}$ PBS and incubated with mouse IgG1 allophycocyanin-conjugated antibody (R\&D Systems; dilution 1:100) and anti-human OV-6 allophycocyanin-conjugated antibody (R\&D Systems; dilution 1:20) for 10 minutes at room temperature and antihuman CD133 allophycocyanin-conjugated antibody (Miltenyi Biotec, San Diego, CA; dilution 1:11) for $30 \mathrm{mi}-$ nutes on ice in the dark. After washing with PBS, cells were resuspended in $1 \mathrm{~mL}$ PBS and examined using the CANTO II fluorescence-activated cell sorting system (Beckton-Dickinson, Franklin Lakes, NJ). Data were analyzed using Kaluza software version 2.1 (Beckman Coulter, Brea, CA).

\section{Verteporfin Treatment for Western Blot Analysis and in Vitro Functional Analysis}

Verteporfin (Sigma-Aldrich) was dissolved in dimethyl sulfoxide (Sigma-Aldrich) and diluted in PBS. The solution was added to the medium at a final concentration of 10 or $20 \mu \mathrm{mol} / \mathrm{L}$ and incubated with cells for 72 hours. An equal concentration of dimethyl sulfoxide was added to the control cells.

\section{Sphere Formation Assay}

HuCCT1 and TKKK cells were seeded in 96-well ultralow attachment plates (Corning Inc., New York, NY) at a density of 10 cells/well in sphere medium. The sphere medium was prepared as previously described. ${ }^{22}$ After culturing cells for 7 days, the number of sphere cells, defined as cell clusters with a diameter $>50 \mu \mathrm{m}$, was counted. The sphere formation rate was assessed as the ratio of the number of spheres on day $7 /$ the number of spheres on day $1 .^{23}$

\section{Anoikis Resistance Assay}

To evaluate the ability of anoikis resistance, HuCCT1 and TKKK cells were incubated with growth factor-free medium for 24 hours at $37^{\circ} \mathrm{C}$, and the colony formation assay was performed. ${ }^{23} \mathrm{~A}$ total of 3000 cells/well suspended in a medium with $0.3 \%$ agar were seeded onto 24 -well culture plates coated with a medium containing $1 \%$ agar (bottom layer). The number of colonies was counted 14 days after seeding cells.

\section{Cytotoxicity Assay}

HuCCT1, TKKK, and HuH-28 cells (3000 cells/well) were seeded onto 96-well plates and precultured for 24 hours. Cell number was quantified after treatment of cells with PBS (as control), verteporfin ( 2 or $5 \mu \mathrm{mol} / \mathrm{L})$, mTOR inhibitor rapamycin ( $25 \mathrm{nmol} / \mathrm{L}$; FUJIFILM Wako Pure Chemical Corp., Osaka, Japan), and the combination of 2 or $5 \mu \mathrm{mol} / \mathrm{L}$ verteporfin and $25 \mathrm{nmol} / \mathrm{L}$ rapamycin using Cell Counting Kit- 8 (Dojindo Laboratories, Kumamoto, Japan) at days 0, 1, 3, and 5.

\section{IL-6-STAT3 Signal Transduction Assay}

Protein expression of STAT3 and p-STAT3 was assessed by Western blot analysis in ICC cell lines. Cells were pretreated with or without $10 \mu \mathrm{mol} / \mathrm{L}$ verteporfin for 2 hours, followed by treatment with or without IL-6 (10 ng/mL; PeproTech Inc., Rocky Hill, NJ) for 20 minutes.

\section{S.C. Injection of Xenograft Model in Vivo Experiments}

All experiments were performed in accordance with animal welfare and the local authorities. Eight-week-old KSN/Slc nude mice (Japan SLC Inc., Hamamatsu, Japan) were used for the in vivo experiments. The cell suspensions, including $1 \times 10^{5} \mathrm{HuCCT} 1$ cells in $50 \mu \mathrm{L}$ of Dulbecco's modified Eagle's medium, were mixed with $50 \mu \mathrm{L}$ of Matrigel (Corning Inc.) and injected subcutaneously into the bilateral flanks of each mouse. Five weeks after cancer cell injection, all mice were blindly divided into three groups (K.S. and M.T.; control group, verteporfin group, and verteporfin and 
A

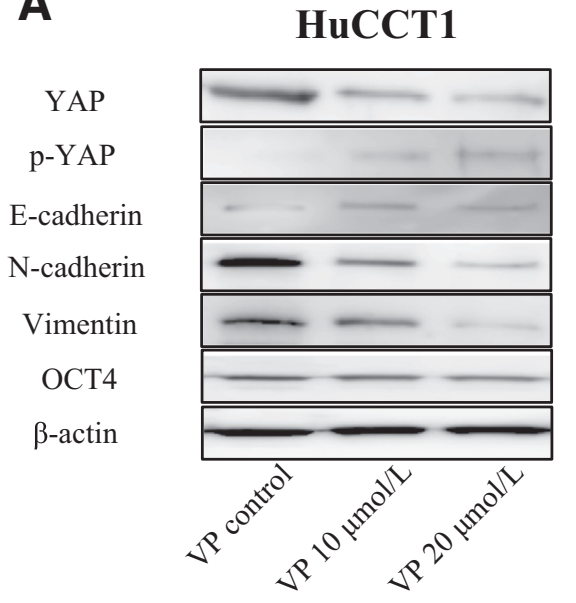

C

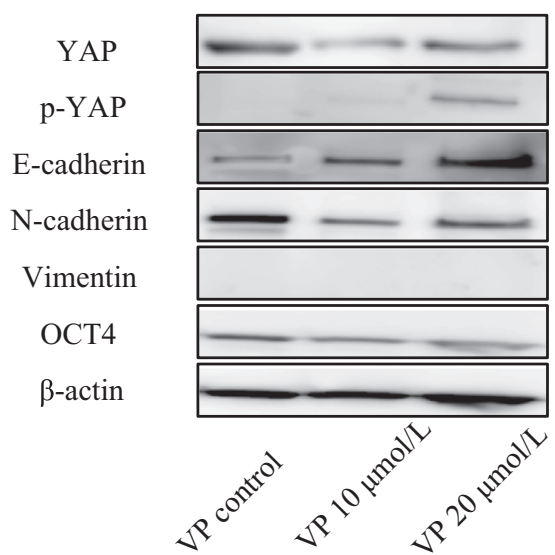

B

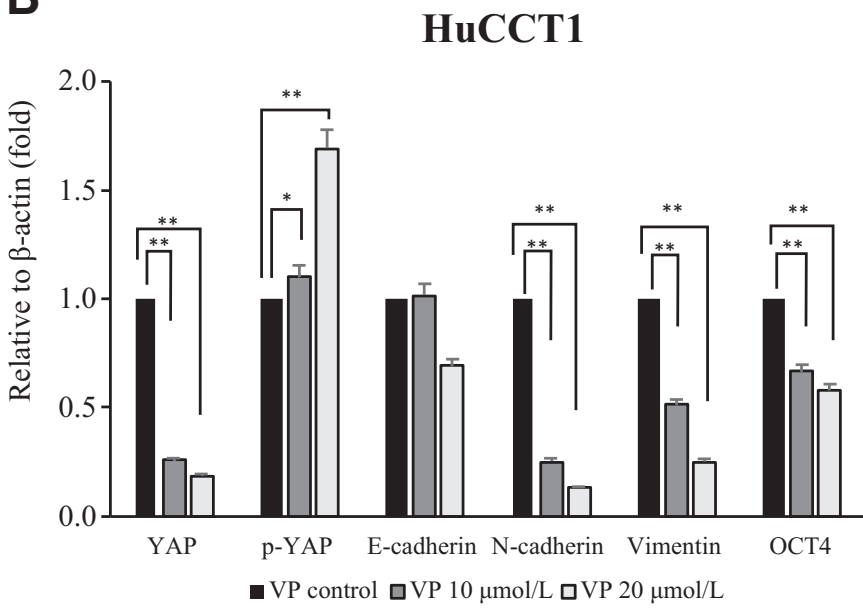

D

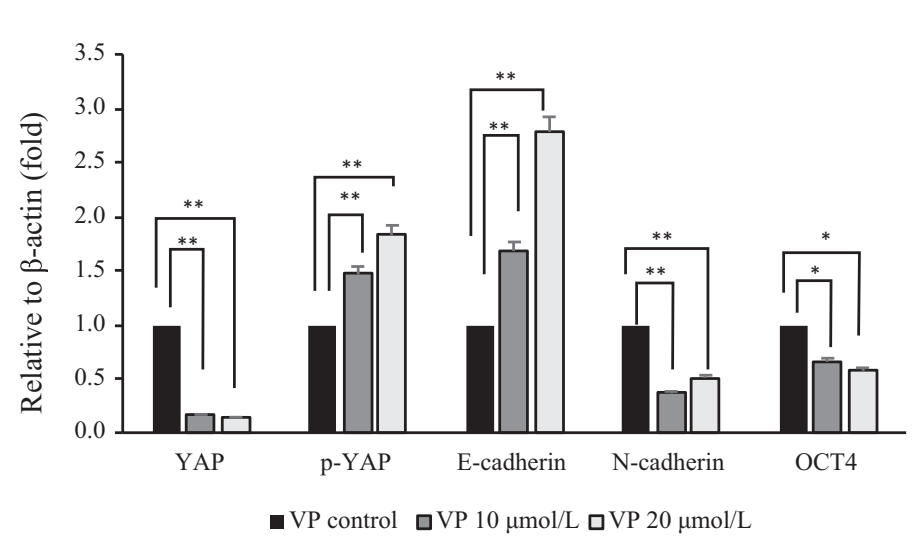

Figure 5 Comparison of the expression of proteins related to epithelial-to-mesenchymal transition/cancer stem cell markers in the intrahepatic cholangiocarcinoma cells treated with or without verteporfin (VP). Expression levels of YAP, phosphorylated YAP (p-YAP), E-cadherin, N-cadherin, vimentin, and OCT4 in HuCCT1 (A) and TKKK (C) cells treated with 10 or $20 \mu \mathrm{mol} / \mathrm{L}$ verteporfin or without verteporfin by Western blot analysis. Comparison of the band intensities of these markers by densitometry in HuCCT1 (B) and TKKK (D) cells. The band intensities were normalized to the intensity of $\beta$-actin. All experiments were repeated at least three times. Data are expressed as means $\pm \mathrm{SEM}$ (B and $\mathbf{D}) .{ }^{*} P<0.05,{ }^{* *} P<0.01$ (Welch $t$-test).

rapamycin group). Xenograft tumors were assessed 3 weeks after initiation of treatment. Animal studies were approved by the Committee on the Use of Live Animals for Teaching and Research of Chiba University.

\section{Statistical Analysis}

Data are expressed as the median \pm SD for all clinical analyses and as the means \pm SEM for the analysis of in vitro experiments. All in vitro experiments were independently assessed at least three times. Tumor volume was calculated using the following formula: $\pi / 6 \times(\mathrm{L} \times \mathrm{W} \times \mathrm{W})$, where $\mathrm{L}$ is the tumor length and $\mathrm{W}$ is the tumor width. ${ }^{23}$ Cumulative survival rates were calculated using the Kaplan-Meier method, and the significance of difference in survival rate was analyzed by the log-rank test. Statistical significance of the results was determined by $U$-test, $\chi^{2}$ test, or Welch $t$-test. $P<0.05$ was considered significant in all analyses. Statistical calculations were performed using JMP 13 (SAS Institute Inc., Cary, NC).

\section{Results}

YAP Expression Is Abundant in Human ICC Tissues and Is Associated with Poor Prognosis

YAP expression was analyzed in human primary ICC tissues with IHC staining. YAP protein was predominantly localized in the nucleus, and weak expression was detected in the cytoplasm of ICC cells (Figure 1A). IHC evaluation revealed 37 cases $(71.2 \%)$ with positive YAP expression in the cell nucleus and 15 cases $(28.8 \%)$ with negative YAP expression among 52 ICC cases (Figure 1, A and B). Analysis of primary tumor volume revealed significantly 
A

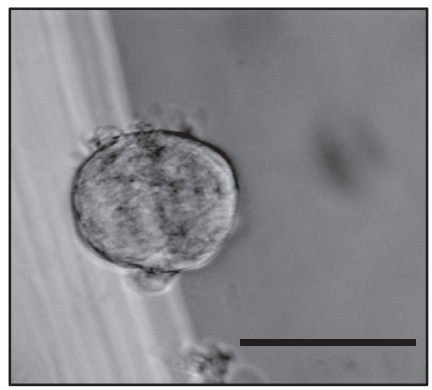

D

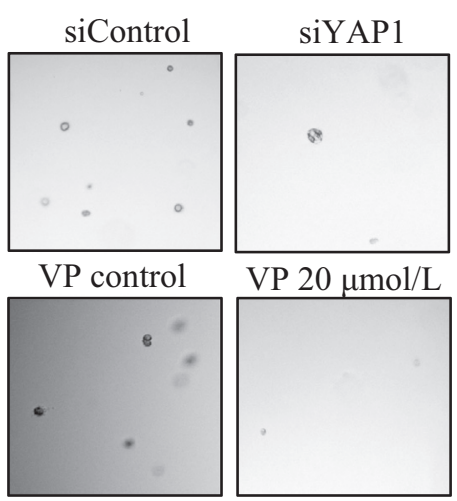

B

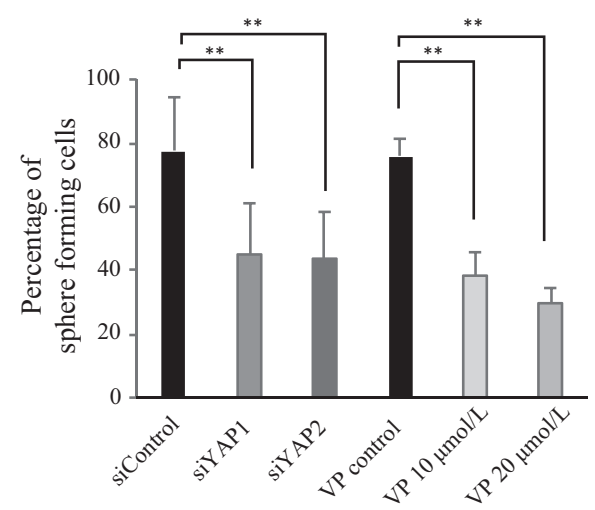

$\mathbf{E}$

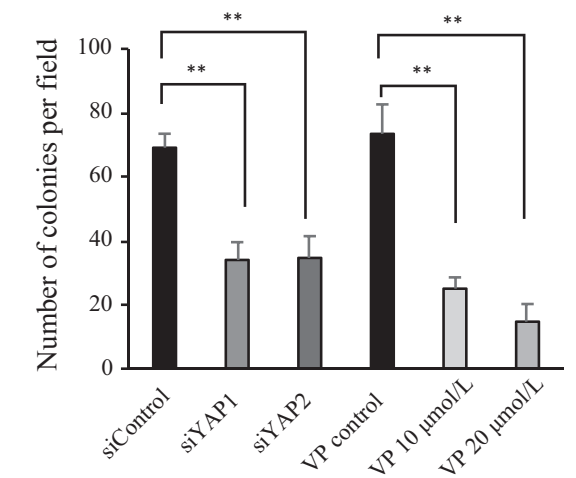

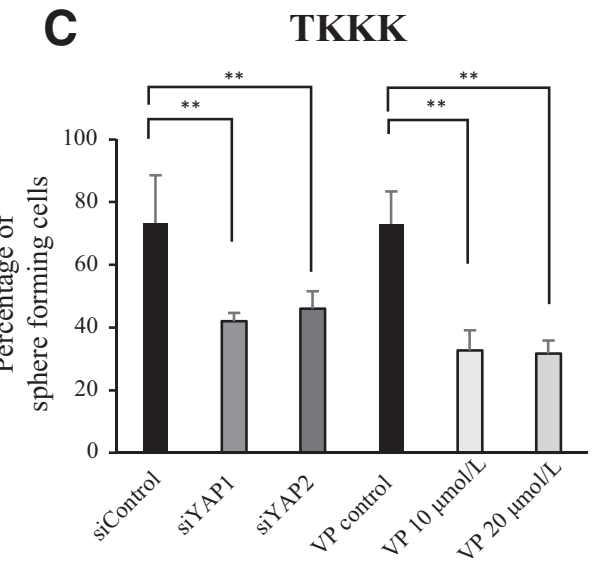

$\mathbf{F}$

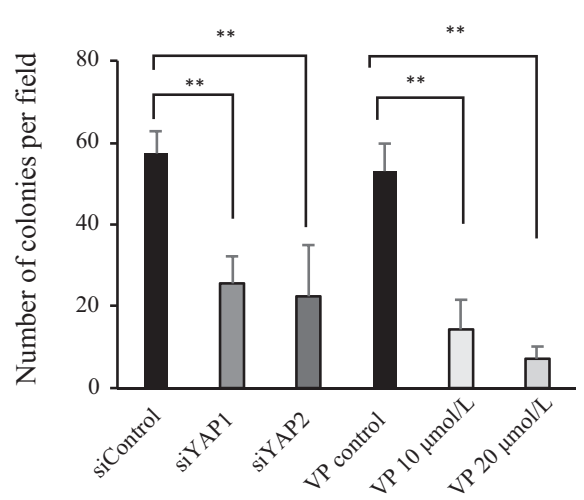

Figure 6 YAP expression maintains the property of cancer stem cell and anoikis resistance in intrahepatic cholangiocarcinoma cells. A: Representative sphere formation in HuCCT1 control cells. B and C: The percentage of sphere-forming cells was evaluated in HuCCT1 (B) and TKKK (C) cells treated with two YAP-specific siRNAs (siYAP1 and siYAP2) and with or without verteporfin (VP; 10 or $20 \mu \mathrm{mol} / \mathrm{L}$ ) treatment. D: Representative colony formation in HuCCT1 cells treated with control siRNA (siControl) or siYAP1 without or with $20 \mu \mathrm{mol} / \mathrm{L}$ verteporfin. $\mathbf{E}$ and $\mathbf{F}$ : The number of colonies was significantly reduced after YAP knockdown and YAP inhibition in HuCCT1 (E) and TKKK (F) cells. All experiments were performed in quadruplicates and repeated at least three times. Data are expressed as means \pm SEM (B, C, E, and $\mathbf{F}$ ). ${ }^{* *} P<0.01$ (Welch $t$-test). Scale bar $=100 \mu \mathrm{m}$ (A). Original magnification, $\times 40$ (D).

larger tumor sizes in the positive YAP expression group than in the negative YAP expression group $(P=0.038)$ (Figure 1C). Clinicopathologic features of groups with positive/negative YAP expression are shown in Table 1. Among various clinical parameters, only primary tumor volume was significantly associated with positive YAP expression $(P=0.02)$. The Kaplan-Meier analysis results showed that the positive YAP group had a significantly shorter overall survival $(P=0.023)$ than the negative YAP group (Figure 1D). Univariate Cox proportional hazard regression analysis revealed significant associations of sex (male), serum carbohydrate antigen $19-9(\geq 36.8 \mathrm{U} / \mathrm{mL})$, primary tumor volume $\left(\geq 34,000 \mathrm{~mm}^{3}\right)$, poor differentiation, presence of para-aortic lymph node metastasis, and positive YAP expression with overall survival (Table 2). These results suggested that positive YAP expression is a good predictor of poor prognosis in patients with ICC after curative surgery.
YAP Expression Positively Correlates with Vimentin, MMP-9, and 0V-6 Expression in Primary ICC

IHC staining for representative EMT markers (E-cadherin, vimentin, and MMP-9) and a CSC marker (OV-6) was performed to investigate the correlation between YAP and EMT/ CSC marker expression in ICC tissues. MMP-9 is a member of the metalloproteinase family involved in the degradation of the extracellular matrix and is known to promote cancer cell invasion. ${ }^{24}$ The epithelial marker OV-6 serves as a marker of oval cell (hepatic progenitor cell of rat) and acts as a human hepatic progenitor cell marker. ${ }^{25}$ The correlations of staining intensities between YAP and these markers are summarized in Figure 2. YAP expression showed a strong negative correlation with E-cadherin expression $(P=0.008)$ (Figure 2, $\mathrm{A}-\mathrm{C}$ ), whereas a positive correlation was observed between YAP expression and vimentin as well as MMP-9 expression (vimentin, $P=0.034$; MMP-9, $P=0.011$ ) (Figure 2, D-I) 
A

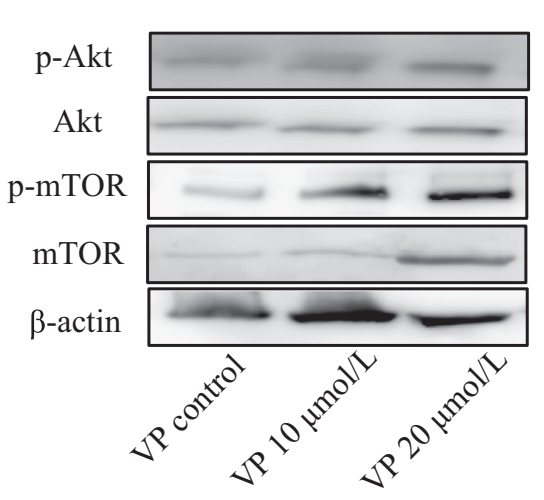

C

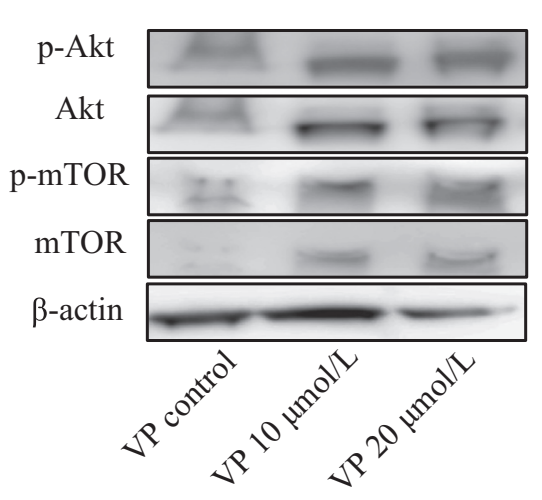

B HuCCT1

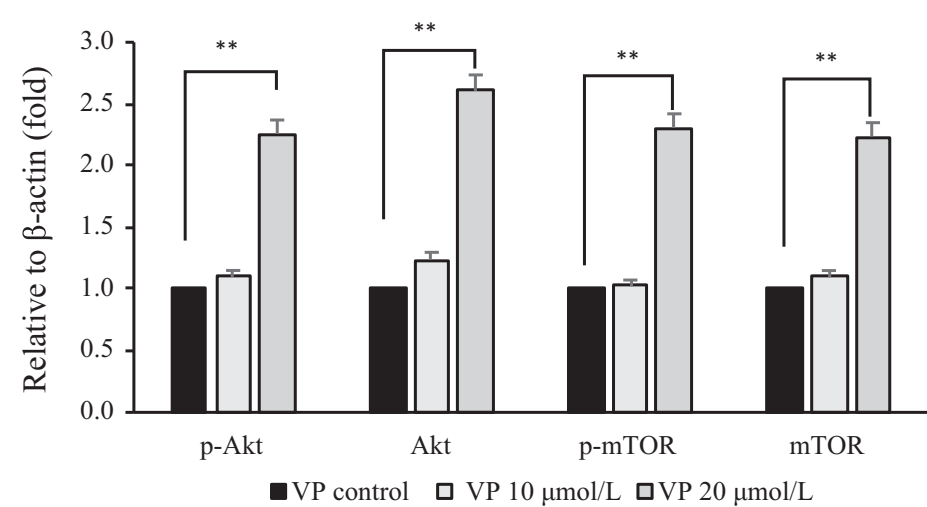

D

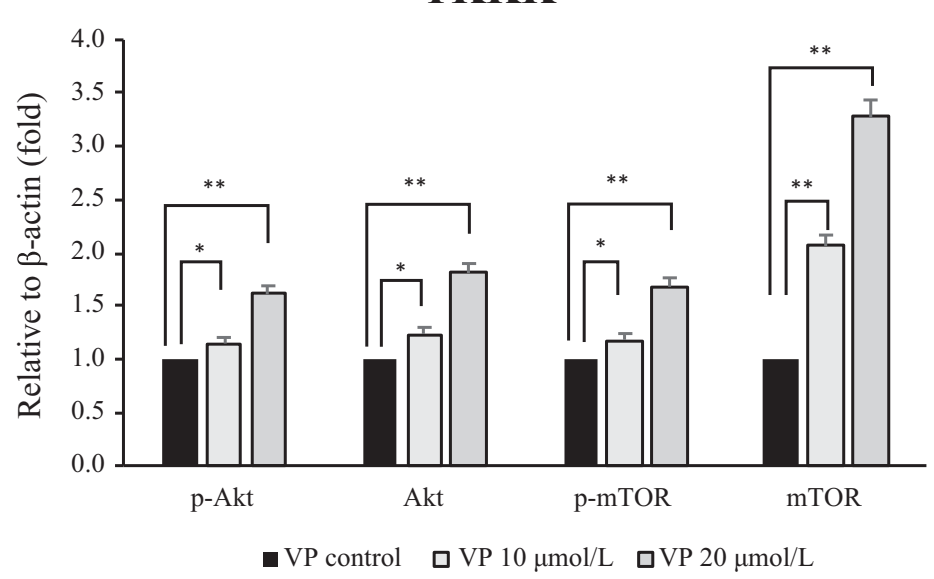

Figure 7 Comparisons of the expression of the proteins related to the Akt/mechanistic target of rapamycin (mTOR) signaling pathway in intrahepatic cholangiocarcinoma cells treated with verteporfin (VP). Expression levels of Akt, phosphorylated Akt (p-Akt), mTOR, and phosphorylated mTOR (p-mTOR) in HuCCT1 (A) and TKKK (C) cells treated with 10 or $20 \mu \mathrm{mol} / \mathrm{L}$ verteporfin or without verteporfin by Western blot analysis. Comparison of the band intensities of these markers by densitometry in HuCCT1 (B) and TKKK (D) cells. The band intensities were normalized to the intensity of $\beta$-actin. All experiments were repeated at least three times. Data are expressed as means $\pm \operatorname{SEM}(\mathbf{B}$ and $\mathbf{D})$. ${ }^{*} P<0.05,{ }^{*} P<0.01$ (Welch $t$-test).

in primary ICC tissues. Furthermore, YAP expression was significantly correlated with OV-6 expression $(P<0.001)$ (Figure 2, J-L). These results implied that YAP expression is closely correlated with mesenchymal and putative CSC property in human ICC.

\section{YAP Expression Is Associated with Expression of Mesenchymal and CSC Markers in ICC Cell Lines}

YAP protein expression was examined in human ICC cell lines by Western blot analysis (Figure 3, A and B). Increased YAP expression was observed in HuCCT1 and TKKK cells, whereas YAP level was low in HuH-28 cells. To confirm the findings of IHC staining, it was examined whether YAP protein expression correlated with EMT and CSC markers in HuCCT1 and TKKK cells using Western blot analysis. In both ICC cell lines, the expression of N- cadherin (mesenchymal marker) and OCT4 (stemness marker) decreased after the knockdown of YAP expression using YAP-specific siRNAs 1 and 2 (Figure 3, C-F). Vimentin expression was also impaired in HuCCT1 cells, whereas E-cadherin level increased after the knockdown of YAP expression in TKKK cells. Next, to further assess the correlation between YAP and CSC features in both ICC cell types, OV-6 and CD133 expression levels in cells in which YAP expression had been knocked down were evaluated by flow cytometry. A significant decrease of the percentage of OV-6 ${ }^{\text {High }}$ and CD133 ${ }^{\text {High }}$ cells in YAP knockdown cells was evident in HuCCT1 cells compared with control cells (Figure 4). Similar results were observed in TKKK cells for OV-6 expression (Supplemental Figure S1). These observations supported the IHC findings and confirmed the association between YAP and mesenchymal and CSC features in ICC cells. 

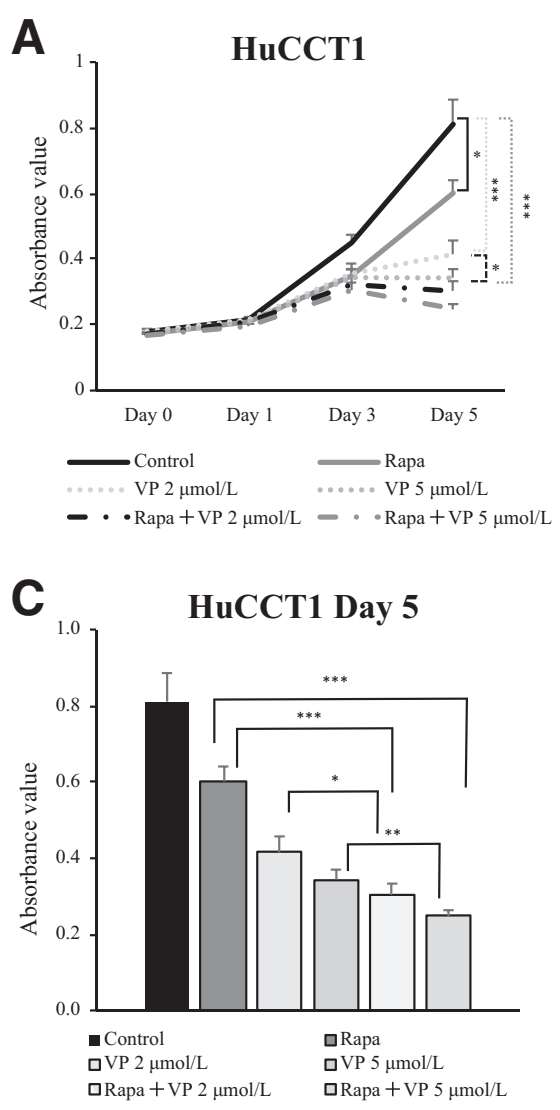

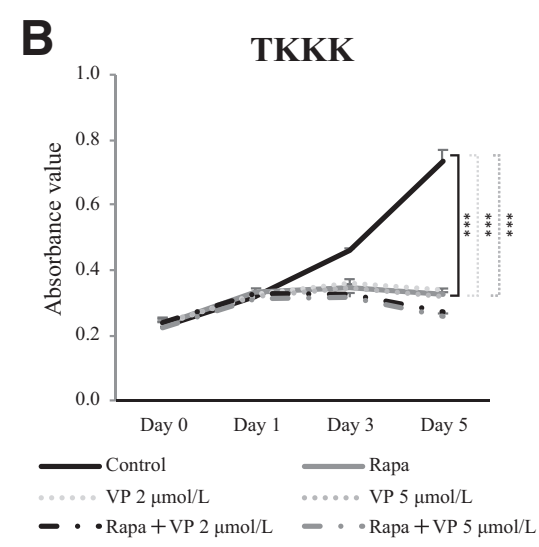

D

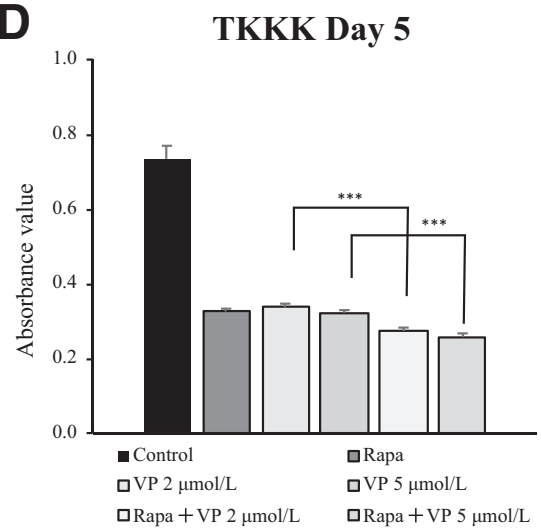

Figure $\mathbf{8}$ Cell cytotoxicity assay in intrahepatic cholangiocarcinoma (ICC) cells. A and $\mathbf{B}$ : The relative number of ICC cells treated with rapamycin (Rapa), 2 or $5 \mu \mathrm{mol} / \mathrm{L}$ verteporfin (VP), and the combination of rapamycin and verteporfin was compared with that of control cells at 0, 1, 3, and 5 days after drug treatment. $\mathbf{C}$ and $\mathbf{D}$ : The results of the combination treatment at 5 days are highlighted. All experiments were independently performed at least three times. Data are expressed as means \pm SEM $(\mathbf{C}$ and $\mathbf{D})$. ${ }^{*} P<0.05$, ${ }^{* *} P<0.01$, and ${ }^{* * *} P<0.001$ (Welch $t$-test).
Verteporfin Decreases YAP Level and Expression of EMT and CSC Markers in ICC Cells

Verteporfin is an inhibitor of YAP expression that is primarily used as a photosensitizer for the treatment of choroidal neovascularization in age-related macular degeneration in ophthalmology. ${ }^{26}$ Verteporfin inhibits YAP activation by preventing its binding to TEAD and suppresses tumor growth in a photodynamic independent manner. ${ }^{27}$ It has been also reported that verteporfin has anticancer effects in several malignancies, including breast, ${ }^{28}$ esophageal, ${ }^{29}$ and pancreatic $^{30}$ cancers. Thus, verteporfin is a candidate as a new target for cancer therapy. ${ }^{31}$ On the basis of these previous results, we hypothesized that the verteporfin-mediated inhibition of YAP expression may result in the regulation of the molecular functions induced by YAP in ICC progression.

The effects of verteporfin treatment were examined on YAP expression in ICC cells by Western blot analysis. Although the expression of total YAP protein decreased, the expression of phosphorylated YAP (p-YAP; inactivated form of YAP) increased in both HuCCT1 and TKKK cells treated with verteporfin compared with control cells (Figure 5). The expression of $\mathrm{N}$-cadherin and vimentin (mesenchymal markers) and OCT4 (CSC marker) decreased in HuCCT1 cells treated with verteporfin. N-cadherin and OCT4 expression also decreased, whereas E-cadherin level increased, in
TKKK cells after treatment with verteporfin (Figure 5). These results indicated that verteporfin inhibits YAP activation and suppresses the mesenchymal and CSC features of ICC cells.

\section{Inhibition of YAP Activation Impairs CSC-Like Property and Anoikis Resistance in ICC Cells}

To evaluate the molecular effects of YAP activation on the property of CSCs, the self-renewal capacity of ICC cells was investigated in vitro with a sphere formation assay (Figure 6A and Supplemental Figure S2A). YAP knocked down or inhibited using two YAP-specific siRNAs or verteporfin, respectively. The treatments significantly decreased the number of sphere-forming cells compared with control HuCCT1 and TKKK cells (Figure 6, B and C). These results indicated that YAP maintains the CSC-like property in ICC cells. Furthermore, it was investigated whether YAP affects anoikis resistance in ICC cells. Anoikis resistance is a major characteristic of CSCs that promotes metastasis. ${ }^{32}$ In the anoikis assay, YAP knockdown and inhibition resulted in a significant decrease in the number and size of colonies compared with the control ICC cells (Figure 6, D-F, and Supplemental Figure S2B), suggesting that YAP contributes to the maintenance of anoikis resistance in ICC cells in vitro. Taken together, these results suggested that YAP is necessary for the 


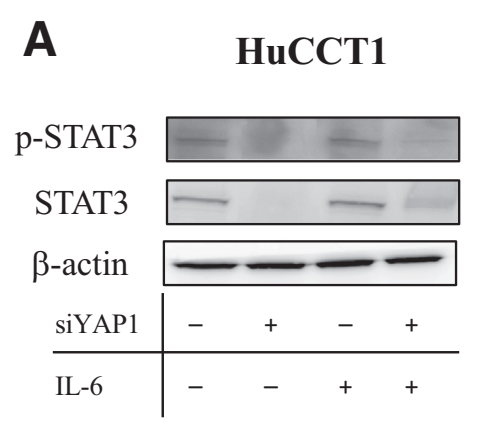

C

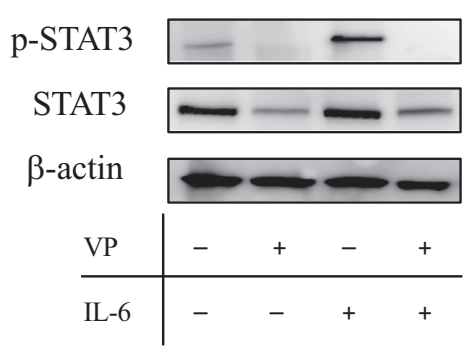

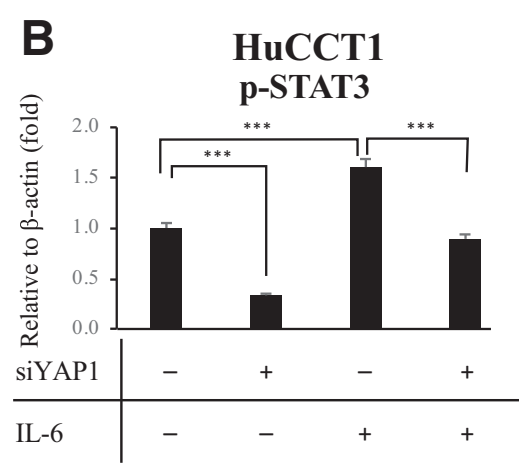

D

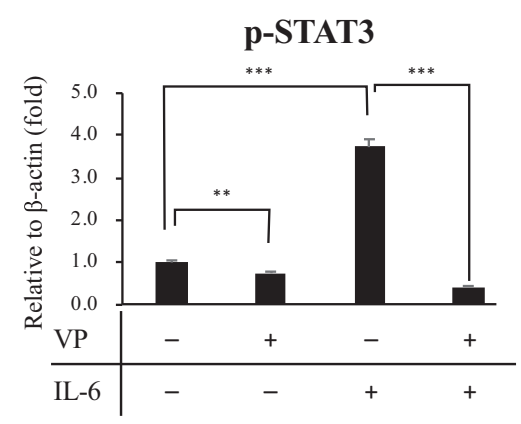

Figure 9 YAP knockdown or verteporfin (VP) inhibits the activation of STAT3 signaling induced by IL- 6 in intrahepatic cholangiocarcinoma cells. HuCCT1 cells were treated with YAP-specific siRNA 1 (siYAP1) or pretreated with phosphate-buffered saline or $5 \mu \mathrm{mol} / \mathrm{L}$ verteporfin for 2 hours, followed by incubation with $10 \mathrm{ng} / \mathrm{mL}$ of IL- 6 for 20 minutes. Expression levels of phosphorylated STAT3 ( $p$ STAT3) and STAT3 in YAP knockdown HuCCT1 cells by YAPspecific siRNA 1 (siYAP1; A) and after verteporfin treatment $(\mathbf{C})$ by Western blot analysis. Comparison of the band intensities of these markers by densitometry in YAP knockdown HuCCT1 cells by siYAP1 (B) and after verteporfin treatment (D). The band intensities were normalized to the intensity of $\beta$-actin. All experiments were performed at least four times. Data are expressed as means \pm SEM (B and D). ${ }^{* *} P<0.01,{ }^{* * *} P<0.001$ (Welch $t$-test). maintenance of CSC characters and facilitates ICC progression.

\section{Combination of Verteporfin and Rapamycin Increases Cytotoxic Effects in ICC Cells}

The activation of the intracellular signal transduction pathway is a crucial event to promote cell survival and growth in response to extracellular signals. The use of a single chemotherapeutic agent, especially selective tyrosine kinase inhibitor, often paradoxically drives the aberrant activation of signaling pathways. ${ }^{33}$ Considering the clinical use of verteporfin for ICC treatment, it is important to understand the signal transduction pathway that is activated or suppressed during verteporfin treatment in ICC cells. Hippo and mTOR are the two major signaling pathways closely involved in the regulation of organ size in Drosophila and mammals. ${ }^{5}$ A previous study demonstrated that the phosphatidylinositol 3-kinase-mTOR pathway is modulated by YAP to regulate cell size, tissue growth, and hyperplasia. ${ }^{34}$ Therefore, the expression of phosphorylated proteins involved in the phosphatidylinositol 3-kinase/mTOR signaling pathway was examined in ICC cells. YAP knockdown by YAP-specific siRNAs did not affect the Akt/mTOR signaling pathway in TKKK cells (Supplemental Figure S3). Contrary to this, both p-Akt and p-mTOR expression levels were significantly up-regulated in HuCCT1 and TKKK cells treated with verteporfin compared with control cells (Figure 7). These results indicated that verteporfin treatment drives the Akt/mTOR signaling transduction pathway in ICC cells.

The cytotoxic effect of verteporfin was investigated on ICC cells. Drug cytotoxicity assay results revealed the significant decrease in the proliferation of HuCCT1 cells after treatment with verteporfin in a dose-dependent manner (Figure 8A). Considering the results of the activated signaling transduction pathway after verteporfin treatment, the cytotoxic effect of the combination of verteporfin and rapamycin, which inhibits the activation of the Akt/mTOR signal transduction in ICC cells, was examined. Additional cytotoxic effects were observed in HuCCT1 and TKKK cells treated with the combination of verteporfin and rapamycin (Figure 8, B-D). Similar results were also observed in HuH-28 cells showing almost no YAP expression (Supplemental Figure S4). These results indicated that the combination of verteporfin and rapamycin inhibits ICC cell proliferation in vitro.

\section{Verteporfin Treatment Impairs IL-6/STAT3 Signal Activation in YAP-Dependent and YAP-Independent Manners}

To understand the mechanism underlying and the cytotoxic effects of YAP signaling and verteporfin treatment through the IL-6/STAT3 signal activation, it was first assessed whether YAP knockdown by the YAP-specific siRNA is involved in IL-6/STAT3 signal activation. YAP knockdown significantly impaired IL-6/STAT3 signal activation in 
A

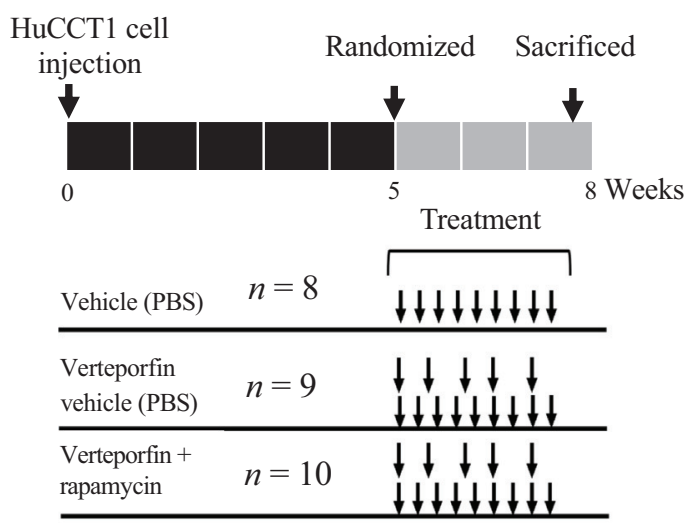

B

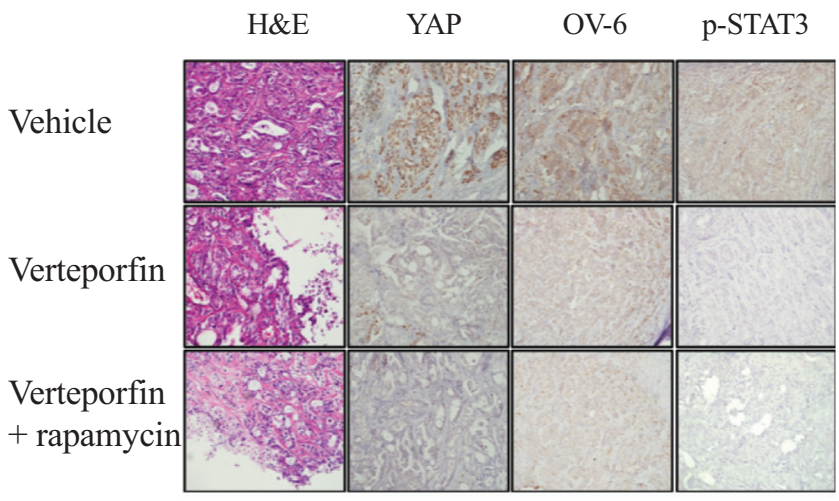

C

D

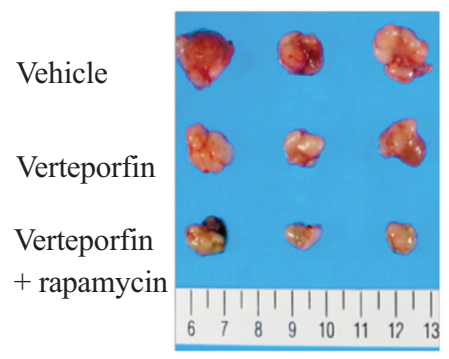

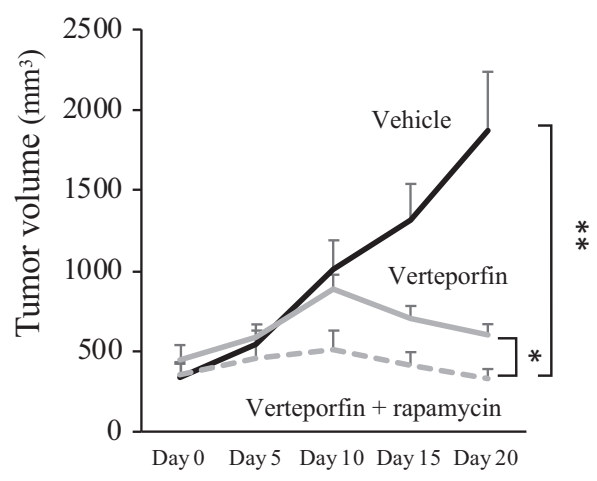

$\mathbf{E}$

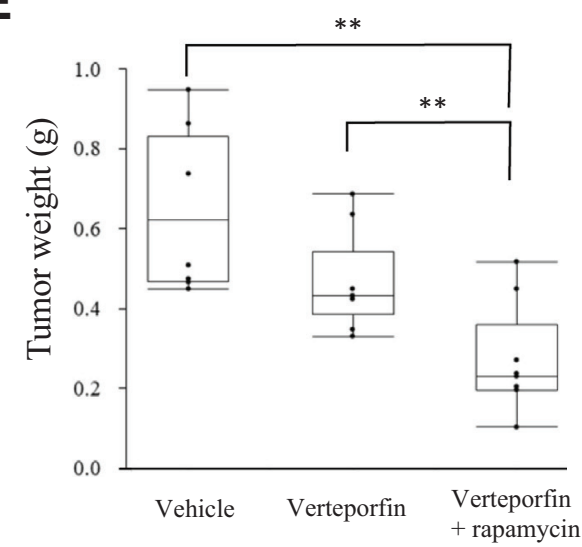

Figure 10 The combination of verteporfin and rapamycin impaired intrahepatic cholangiocarcinoma (ICC) tumor growth in a s.c. xenograft model. A: Experimental design of the randomized trial using HuCTT1 cells in a s.c. injection model. Vehicle [phosphate-buffered saline (PBS)] control, verteporfin, and the combination of verteporfin with rapamycin were administrated through i.p. injection. B: Representative histology of ICC xenograft tumors of control group (vehicle), verteporfin group, and combination therapy group. Hematoxylin and eosin (H\&E) staining and immunohistochemistry for YAP, OV-6, and phosphorylated STAT3 (p-STAT3) in tissues of the three experimental groups. C: Representative macroscopic findings of tumors among each group. D and E: Tumor volumes (D) and tumor weights (E) are depicted for each treatment group in line graph and box-plot histograms, respectively. Data are expressed as means \pm SEM (D). ${ }^{*} P<0.05,{ }^{*} P<0.01$ (Welch $t$-test). Original magnification, $\times 200$ (B).

HuCCT1 cells (Figure 9, A and B). Next, p-STAT3 expression was investigated after the exposure of verteporfin-treated ICC cells to IL-6. Similar to the previous reports on basal-like breast cancer cells ${ }^{21}$ and colon cancer cells, ${ }^{35}$ IL-6-stimulated STAT3 phosphorylation was impaired after treatment of ICC cells with verteporfin in HuCCT1 (Figure 9, C and D). In addition, the activation of IL-6/STAT3 signaling was inhibited after verteporfin treatment in low YAP-expressing HuH-28 cells (Supplemental Figure S5). Taken together, these results indicated that verteporfin induces cytotoxic effects by regulating multiple signaling pathways, including the IL-6/STAT3 pathway, in YAPdependent and YAP-independent manners in ICC cells.

\section{Combination of Verteporfin and Rapamycin Impairs ICC} Tumor Growth in the S.C. Xenograft Model

Last, s.c. injection of HuCCT1 cells in mice was performed to investigate whether the combination of verteporfin and rapamycin diminished ICC tumor growth in a preclinical in vivo experiment (Figure 10A). Immunostaining for YAP, OV-6, and p-STAT3 revealed decreased staining intensities in the combination group compared with the control group (Figure 10B). Notably, the combination therapy significantly reduced both the volume and the weight of tumors in comparison with the control group or the verteporfin alone group (Figure 10, C-E). Together, these data indicated that targeting both YAP and Akt/mTOR signaling may offer a new therapeutic approach in ICC tumors.

\section{Discussion}

In the present study, we demonstrate that the positive YAP expression in human ICC tissues correlates with the expression of EMT and CSC markers and is significantly associated with poor prognosis of patients with ICC. YAP contributes to the maintenance of cancer stemness and anoikis resistance in ICC cells. The combination of verteporfin and rapamycin had additional cytotoxic effects on 


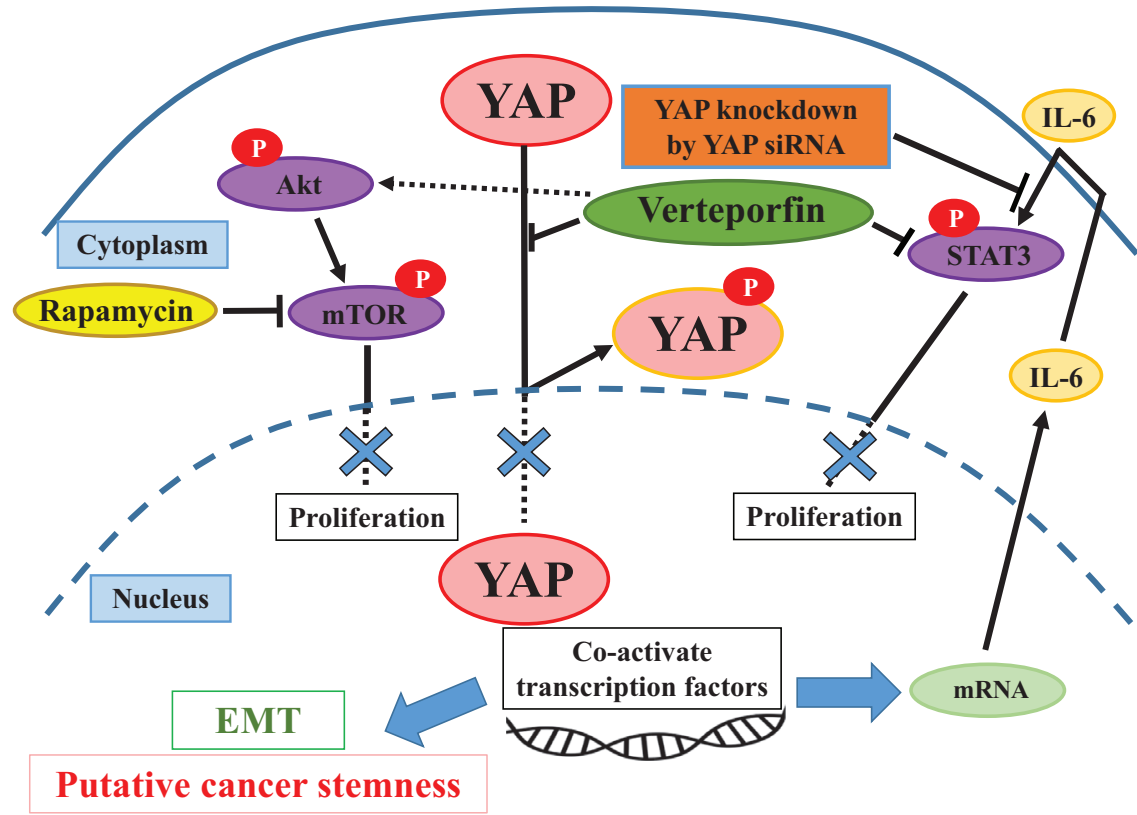

Figure 11 Scheme of the relationship between YAP activation and multiple verteporfin effects on intracellular signaling pathway in intrahepatic cholangiocarcinoma cells. Verteporfin inhibits activation of YAP, whereas verteporfin up-regulates $\mathrm{p}-\mathrm{Akt} / \mathrm{p}$-mTOR expression levels. YAP knockdown or verteporfin inhibits the activation of STAT3 signaling induced by IL-6. Akt, A serine/ threonine kinase; EMT, epithelial-to-mesenchymal transition; mTOR, mechanistic target of rapamycin; $\mathrm{P}$, phosphorylated; STAT3, signal transducer and activator of transcription 3.
ICC both in vitro and in vivo. These results reveal a novel function of YAP that is correlated with the property of cancer stemness. These observations suggest a novel therapeutic strategy for ICC treatment (Figure 11).

YAP is considered an essential protein for organ size regulation ${ }^{8}$; and it acts as a crucial component of the Hippo/ YAP signaling pathway, regulating cellular proliferation in response to cell contact. ${ }^{9}$ Recent studies have suggested the potent role of Hippo/YAP signaling in controlling liver cell fate and phenotypic plasticity in mature hepatocytes. ${ }^{36,37}$ The functional roles of YAP in neoplastic cells vary; they may include proliferation, invasion, and metastasis. Activation of YAP expression has been associated with poor prognosis in various human malignancies. ${ }^{10-14}$ EMT promotes cancer progression through a variety of mechanisms and endows cells with migratory and invasive properties. In addition, EMT induces CSC properties that initiate and maintain tumor growth. ${ }^{38} \mathrm{~A}$ previous study reported that YAP promotes cell proliferation, chemoresistance, and angiogenesis through TEAD in ICC. ${ }^{18}$ In esophageal cancer, YAP-driven expression of sex-determining region $\mathrm{Y}$ box 9 is a critical event in the acquisition of CSC properties, suggesting that YAP inhibition may be an effective therapeutic approach to target CSC population. ${ }^{29}$ This study reveals the relationship between YAP expression and EMT and CSC features in human ICC tissues, and it highlights the functions of CSC in ICC cells. A positive correlation was observed between expression of YAP and EMT/CSC markers. YAP has a pivotal role in the maintenance of CSC properties in ICC cells.

It is crucial to elucidate the mechanism underlying YAPmediated regulation of ICC cells. YAP overexpression or knockdown can increase or decrease p-Akt levels, respectively, in ICC cells. ${ }^{17}$ In the present study, YAP knockdown with YAP-specific siRNAs did not affect the activation of the Akt/mTOR signaling pathway in ICC cells. The activation of the intracellular signal transduction often affects other signaling pathways via the compensatory signaling mechanism or feedback loop. ${ }^{33}$ Emerging evidence from previous studies suggests multiple effects of the YAP inhibitor, verteporfin, on various signaling pathways in cancers. Liu-Chittenden et $\mathrm{al}^{27}$ found that verteporfin disrupts the formation of the YAP-TEAD complex by binding to YAP and changes its conformation, thereby blocking the transcription of downstream targets. Moreover, Wang et al ${ }^{39}$ recently demonstrated that YAP drives the self-renewal of OV-6-positive CSCs to facilitate cancer progression via the YAP/TEAD1/platelet-derived growth factor-BB/plateletderived growth factor receptor loop in bladder cancer. Thus, the anticancer effects of verteporfin are mediated by various molecular mechanisms in several malignancies. ${ }^{26,30,35}$ A previous study described that verteporfin down-regulates YAP and p-YAP expression and suppresses p-Akt levels in endometrial cancer cells. ${ }^{40}$ On the contrary, increases were observed in p-YAP expression and activation of the Akt/mTOR signaling pathway after inhibition of YAP by verteporfin. The mechanism underlying the activation of the Akt/mTOR signaling pathway, mediated via YAP in ICC, is still unclear. Verteporfin likely drives the aberrant activation of Akt/mTOR signaling in a compensatory system. These paradoxical results after YAP knockdown in ICC cells suggest that verteporfin not only affects YAP or pYAP expression but also influences multiple signaling pathways in ICC cells. Kim et al ${ }^{21}$ demonstrated the critical role of the serum response factor-IL-6 axis in YAP-induced stemness in mammary epithelial cells and breast cancer. Consistent with this observation, YAP knockdown or verteporfin treatment dramatically blocked the IL-6-induced 
STAT3 signal transduction in ICC cells. Zhang et $\mathrm{al}^{35}$ recently showed that verteporfin treatment induces tumorspecific proteotoxicity and inhibits colorectal cancer progression in a mouse model via the IL-6/STAT3 pathway in a YAP-independent manner. Consistent with this finding, verteporfin treatment significantly inhibited the proliferation of all three ICC cell lines, including cells with low expression of YAP protein. The results demonstrate, for the first time, that the combination of verteporfin and mTOR inhibitor has additional cytotoxicity for ICC in vitro and in vivo. These results suggest that verteporfin inhibits the translocation of YAP into the cell nucleus and plays a role in the regulation of antiproliferative ability in YAP-dependent and YAP-independent manners in ICC cells.

In conclusion, YAP maintains putative cancer stemness properties and is associated with poor prognosis in ICC. This study provides the first demonstration of the role of YAP in the promotion of CSC properties in ICC and shows that the use of the combination of verteporfin and mTOR inhibitor may serve as a novel strategy for ICC treatment. Future clinical studies are warranted to determine whether the inhibition of YAP and Akt/mTOR signaling affects the control of ICC progression.

\section{Acknowledgments}

We thank Drs. Kensuke Suzuki, Hitoe Nishino, Shinichiro Nakada, and Naoko Yoneura (Chiba University, Japan) for the technical support in experiments.

S.T. and M.O. are the guarantors of this work and, as such, had full access to all the data in the study and take full responsibility for the integrity of the data and the accuracy of the data analysis.

\section{Supplemental Data}

Supplemental material for this article can be found at http://doi.org/10.1016/j.ajpath.2019.05.014.

\section{References}

1. Malhi H, Gores GJ: Cholangiocarcinoma: modern advances in understanding a deadly old disease. J Hepatol 2006, 45:856-867

2. Rizvi S, Gores GJ: Pathogenesis, diagnosis, and management of cholangiocarcinoma. Gastroenterology 2013, 145:1215-1229

3. Buettner S, van Vugt JL, IJzermans JN, Groot Koerkamp B: Intrahepatic cholangiocarcinoma: current perspectives. Onco Targets Ther 2017, 10:1131-1142

4. Lee K, Lee KB, Jung HY, Yi NJ, Lee KW, Suh KS, Jang JJ: The correlation between poor prognosis and increased yes-associated protein 1 expression in keratin 19 expressing hepatocellular carcinomas and cholangiocarcinomas. BMC Cancer 2017, 17:441

5. Dong J, Feldmann G, Huang J, Wu S, Zhang N, Comerford SA, Gayyed MF, Anders RA, Maitra A, Pan D: Elucidation of a universal size-control mechanism in Drosophila and mammals. Cell 2007, 130: $1120-1133$

6. Pan D: The hippo signaling pathway in development and cancer. Dev Cell 2010, 19:491-505
7. Gao T, Zhou D, Yang C, Singh T, Penzo-Mendez A, Maddipati R, Tzatsos A, Bardeesy N, Avruch J, Stanger BZ: Hippo signaling regulates differentiation and maintenance in the exocrine pancreas. Gastroenterology 2013, 144:1543-1553. 1553.e1

8. Mo JS, Park HW, Guan KL: The Hippo signaling pathway in stem cell biology and cancer. EMBO Rep 2014, 15:642-656

9. Zhao B, Wei X, Li W, Udan RS, Yang Q, Kim J, Xie J, Ikenoue T, Yu J, Li L, Zheng P, Ye K, Chinnaiyan A, Halder G, Lai ZC, Guan KL: Inactivation of YAP oncoprotein by the Hippo pathway is involved in cell contact inhibition and tissue growth control. Genes Dev 2007, 21:2747-2761

10. Xu MZ, Yao TJ, Lee NP, Ng IO, Chan YT, Zender L, Lowe SW, Poon RT, Luk JM: Yes-associated protein is an independent prognostic marker in hepatocellular carcinoma. Cancer 2009, 115: 4576-4585

11. Hall CA, Wang R, Miao J, Oliva E, Shen X, Wheeler $T$, Hilsenbeck SG, Orsulic S, Goode S: Hippo pathway effector Yap is an ovarian cancer oncogene. Cancer Res 2010, 70: $8517-8525$

12. Wang L, Shi S, Guo Z, Zhang X, Han S, Yang A, Wen W, Zhu Q: Overexpression of YAP and TAZ is an independent predictor of prognosis in colorectal cancer and related to the proliferation and metastasis of colon cancer cells. PLoS One 2013, 8:e65539

13. Kang W, Tong JH, Chan AW, Lee TL, Lung RW, Leung PP, So KK, Wu K, Fan D, Yu J, Sung JJ, To KF: Yes-associated protein 1 exhibits oncogenic property in gastric cancer and its nuclear accumulation associates with poor prognosis. Clin Cancer Res 2011, 17: 2130-2139

14. Li M, Lu J, Zhang F, Li H, Zhang B, Wu X, Tan Z, Zhang L, Gao G, Mu J, Shu Y, Bao R, Ding Q, Wu W, Dong P, Gu J, Liu Y: Yesassociated protein 1 (YAP1) promotes human gallbladder tumor growth via activation of the AXL/MAPK pathway. Cancer Lett 2014, 355:201-209

15. Avruch J, Zhou D, Fitamant J, Bardeesy N, Mou F, Barrufet LR: Protein kinases of the Hippo pathway: regulation and substrates. Semin Cell Dev Biol 2012, 23:770-784

16. Marti P, Stein C, Blumer T, Abraham Y, Dill MT, Pikiolek M, Orsini V, Jurisic G, Megel P, Makowska Z, Agarinis C, Tornillo L, Bouwmeester T, Ruffner H, Bauer A, Parker CN, Schmelzle T, Terracciano LM, Heim MH, Tchorz JS: YAP promotes proliferation, chemoresistance, and angiogenesis in human cholangiocarcinoma through TEAD transcription factors. Hepatology 2015, 62: $1497-1510$

17. Pei T, Li Y, Wang J, Wang H, Liang Y, Shi H, Sun B, Yin D, Sun J, Song R, Pan S, Sun Y, Jiang H, Zheng T, Liu L: YAP is a critical oncogene in human cholangiocarcinoma. Oncotarget 2015, 6: $17206-17220$

18. Anakk S, Bhosale M, Schmidt VA, Johnson RL, Finegold MJ, Moore DD: Bile acids activate YAP to promote liver carcinogenesis. Cell Rep 2013, 5:1060-1069

19. Du L, Li YJ, Fakih M, Wiatrek RL, Duldulao M, Chen Z, Chu P, Garcia-Aguilar J, Chen Y: Role of SUMO activating enzyme in cancer stem cell maintenance and self-renewal. Nat Commun 2016, 7:12326

20. Zhao AY, Dai YJ, Lian JF, Huang Y, Lin JG, Dai YB, Xu TW: YAP regulates ALDH1A1 expression and stem cell property of bladder cancer cells. Onco Targets Ther 2018, 11:6657-6663

21. Kim T, Yang SJ, Hwang D, Song J, Kim M, Kyum Kim S, Kang K, Ahn J, Lee D, Kim MY, Kim S, Seung Koo J, Seok Koh S, Kim SY, Lim DS: A basal-like breast cancer-specific role for SRF-IL6 in YAPinduced cancer stemness. Nat Commun 2015, 6:10186

22. Rovira M, Scott SG, Liss AS, Jensen J, Thayer SP, Leach SD: Isolation and characterization of centroacinar/terminal ductal progenitor cells in adult mouse pancreas. Proc Natl Acad Sci U S A 2010, 107:75-80

23. Takano S, Reichert M, Bakir B, Das KK, Nishida T, Miyazaki M, Heeg S, Collins MA, Marchand B, Hicks PD, Maitra A, Rustgi AK: 
Prrx1 isoform switching regulates pancreatic cancer invasion and metastatic colonization. Genes Dev 2016, 30:233-247

24. Davey A, McAuley DF, O'Kane CM: Matrix metalloproteinases in acute lung injury: mediators of injury and drivers of repair. Eur Respir J 2011, 38:959-970

25. Yang W, Yan HX, Chen L, Liu Q, He YQ, Yu LX, Zhang SH, Huang DD, Tang L, Kong XN, Chen C, Liu SQ, Wu MC, Wang HY: Wnt/beta-catenin signaling contributes to activation of normal and tumorigenic liver progenitor cells. Cancer Res 2008, 68:4287-4295

26. Michels S, Schmidt-Erfurth U: Photodynamic therapy with verteporfin: a new treatment in ophthalmology. Semin Ophthalmol 2001, 16:201-206

27. Liu-Chittenden Y, Huang B, Shim JS, Chen Q, Lee SJ, Anders RA, Liu JO, Pan D: Genetic and pharmacological disruption of the TEADYAP complex suppresses the oncogenic activity of YAP. Genes Dev 2012, 26:1300-1305

28. Lin CH, Pelissier FA, Zhang H, Lakins J, Weaver VM, Park C, LaBarge MA: Microenvironment rigidity modulates responses to the HER2 receptor tyrosine kinase inhibitor lapatinib via YAP and TAZ transcription factors. Mol Biol Cell 2015, 26:3946-3953

29. Song S, Ajani JA, Honjo S, Maru DM, Chen Q, Scott AW, Heallen TR, Xiao L, Hofstetter WL, Weston B, Lee JH, Wadhwa R, Sudo K, Stroehlein JR, Martin JF, Hung MC, Johnson RL: Hippo coactivator YAP1 upregulates SOX9 and endows esophageal cancer cells with stem-like properties. Cancer Res 2014, 74: $4170-4182$

30. Donohue E, Thomas A, Maurer N, Manisali I, Zeisser-Labouebe M, Zisman N, Anderson HJ, Ng SS, Webb M, Bally M, Roberge M: The autophagy inhibitor verteporfin moderately enhances the antitumor activity of gemcitabine in a pancreatic ductal adenocarcinoma model. J Cancer 2013, 4:585-596

31. Stanger BZ: Quit your YAPing: a new target for cancer therapy. Genes Dev 2012, 26:1263-1267

32. Schmidt JM, Panzilius E, Bartsch HS, Irmler M, Beckers J, Kari V, Linnemann JR, Dragoi D, Hirschi B, Kloos UJ, Sass S, Theis F,
Kahlert S, Johnsen SA, Sotlar K, Scheel CH: Stem-cell-like properties and epithelial plasticity arise as stable traits after transient Twist1 activation. Cell Rep 2015, 10:131-139

33. Logue JS, Morrison DK: Complexity in the signaling network: insights from the use of targeted inhibitors in cancer therapy. Genes Dev 2012, 26:641-650

34. Tumaneng K, Schlegelmilch K, Russell RC, Yimlamai D, Basnet H, Mahadevan N, Fitamant J, Bardeesy N, Camargo FD, Guan KL: YAP mediates crosstalk between the Hippo and PI(3)K-TOR pathways by suppressing PTEN via miR-29. Nat Cell Biol 2012, 14:1322-1329

35. Zhang H, Ramakrishnan SK, Triner D, Centofanti B, Maitra D, Gyorffy B, Sebolt-Leopold JS, Dame MK, Varani J, Brenner DE, Fearon ER, Omary MB, Shah YM: Tumor-selective proteotoxicity of verteporfin inhibits colon cancer progression independently of YAP1. Sci Signal 2015, 8:ra98

36. Yimlamai D, Christodoulou C, Galli GG, Yanger K, Pepe-Mooney B, Gurung B, Shrestha K, Cahan P, Stanger BZ, Camargo FD: Hippo pathway activity influences liver cell fate. Cell 2014, 157:1324-1338

37. Miyamura N, Hata S, Itoh T, Tanaka M, Nishio M, Itoh M, Ogawa $Y$, Terai S, Sakaida I, Suzuki A, Miyajima A, Nishina H: YAP determines the cell fate of injured mouse hepatocytes in vivo. Nat Commun 2017, 8:16017

38. Thiery JP, Acloque H, Huang RY, Nieto MA: Epithelial-mesenchymal transitions in development and disease. Cell 2009, 139: $871-890$

39. Wang KJ, Wang C, Dai LH, Yang J, Huang H, Ma X, Zhou Z, Yang ZY, Xu W, Hua MM, Lu X, Zeng S, Wang HQ, Zhang Z, Cheng YQ, Liu D, Tian QQ, Sun YH, Xu CL: Targeting an autocrine regulatory loop in cancer stem-like cells impairs the progression and chemotherapy resistance of bladder cancer. Clin Cancer Res 2019, 25:1070-1086

40. Dasari VR, Mazack V, Feng W, Nash J, Carey DJ, Gogoi R: Verteporfin exhibits YAP-independent anti-proliferative and cytotoxic effects in endometrial cancer cells. Oncotarget 2017, 8: $28628-28640$ 